\title{
GLASSCHALEN VOM TYP WEKLICE AUS DEN GRÄBERN 110 UND 211 DES SPÄTKAISERZEIT LICH-/ FRÜHVÖLKERWANDERUNGSZEITLICHEN GRÄBERFELDES VOJTENKI 1
}

\author{
Vladyslav Shchepachenko
}

DOI: https://doi.org/10.31577/szausav.2020.67.4

Keywords: Eastern Europe, Eastern Ukraine, Late Roman time, Chernyakhov culture, glass vessels, glass bowls type Weklice

\begin{abstract}
Glass bowls of Weklice type from graves 110 and 211 of Vojtenki 1 Late Roman - Early Great migration period burial ground

Current publication is devoted to the injection of two same-type glass bowls from Cherniakhiv culture burial ground into the circle of scientific knowledge. Similar glass bowls are came from graves 110 and 211 on Vojtenki 1 burial ground (Eastern Ukraine). During the research, presumable closest analogies to them were found. These analogies are vessels from Weklice, Poland (burial 82) and Danceni, Republic Moldova (burial 169). T. Stawiarska was identified them as "Weklice" type. Distinctive and peculiar morphological features of each of the bowls indicate that the Weklice type vessels are unlikely to have a common origin. However, despite these differences, similar shape, the same techniques for processing a rim and a similar ornament composition allow us to rank these bowls to type of product.

Some researchers have associated "hot decoration" way of glassware production with the provincial-Roman glassworking technology. That is why, it could be assumed, that Weklice type glass bowls have provincial-Roman origin. The evolution of the glassware forms in Roman world allows us to establish the time of their production. It is limited to the period between the second third of the $3^{\text {rd }}$ century - the end of $3^{\text {rd }} /$ beginning of $4^{\text {th }}$ centuries. Lower chronological frame corresponds to the time of appearance of such products in Barbaricum, and the upper one to the time of disappearance of glassware with fire-rounded rim in a number of border provinces of the Empire. Nevertheless, the dating of burials 110 and 211 from Vojtenki 1, shows that such products continue to exist in Barbaricum for a rather long time and fall into complexes at least at chronological stage C3. At the Voitenki 1 burial ground, such bowls are found only in high or special social status female graves. Moreover, the same type of vessel appears in burials with different burial rite as a grave good.
\end{abstract}

In letzten zwei Jahrzehnten beobachten wir ein wachsendes Interesse am Studium von Glasgefäßen aus dem Gebiet der Sântana de Mureş/Černjachon-Kultur. Davon zeugt die große Zahl der Publikationen zu dem Thema, die innerhalb dieses Zeitraumes veröffentlicht wurden: Untersuchungen zur Provenienz und zur Herstellungstechnologie (Rumyantseva 2016, 299-315; 2017a, 141; 2017b, 193-199; Rumyantseva/Belikov 2017, 257-264; Rumyantseva/Lyubichev/Trifonov 2018, 182-208; Rumyantseva/Shcherbakov 2016, 203-217), zur Entwicklung der Typologie und Präzisierung der Chronologie von Glasgefäßen (Gavritukhin 1999, 48-86; 2017, 83-109; Petrauskas 2016, 89-106; 2017, 123-154) und zur Einführung neuer Materialien in den wissenschaftlichen Diskurs (Petrauskas/Pasternak 2003, 65-74; Reida/Heiko/Sapiehin 2018, 111-120). In Verbindung damit bleiben die Publikation neuer Formen von Glasgefäßen und die Bestimmung des Platzes dieser Gefäße innerhalb der existierenden typologischen und chronologischen Systeme in Verbindung mit der Rekonstruktion kultureller und historischer Prozesse in der Region aktuell.

Diese Publikation ${ }^{1}$ dient der Einführung zweier Schalen eines Typs aus den Gräbern 110 und 211 des Gräberfeldes Vojtenki 1 in die wissenschaftliche Diskussion. ${ }^{2}$

1 Mein Dank gilt Prof. M. V. Ljubičev für die Möglichkeit, unveröffentlichte Materialien des Gräberfeldes Vojtenki zu nutzen und Dr. Vlad Vornic für das Foto einer Schale aus dem Grab 169 des Gräberfeldes Dănceni. Mein Dank gilt Dr. E. Schultze und Prof. M. V. Ljubičev für die Übersetzung dieses Artikels ins Deutsche. Mein Dank gilt Dr. E. Droberjar für die Hilfe beim Veröffentlichen dieses Artikels.

2 Das Gräberfeld Vojtenki 1 ist Bestandteil eines archäologischen Komplexes der Späten Römischen Kaiserzeit - Anfang der Völkerwanderungszeit und gehört zu einer Siedlung, die sich am Abhang zu einem kleinen Fluss (heute ein Teich) sechster 


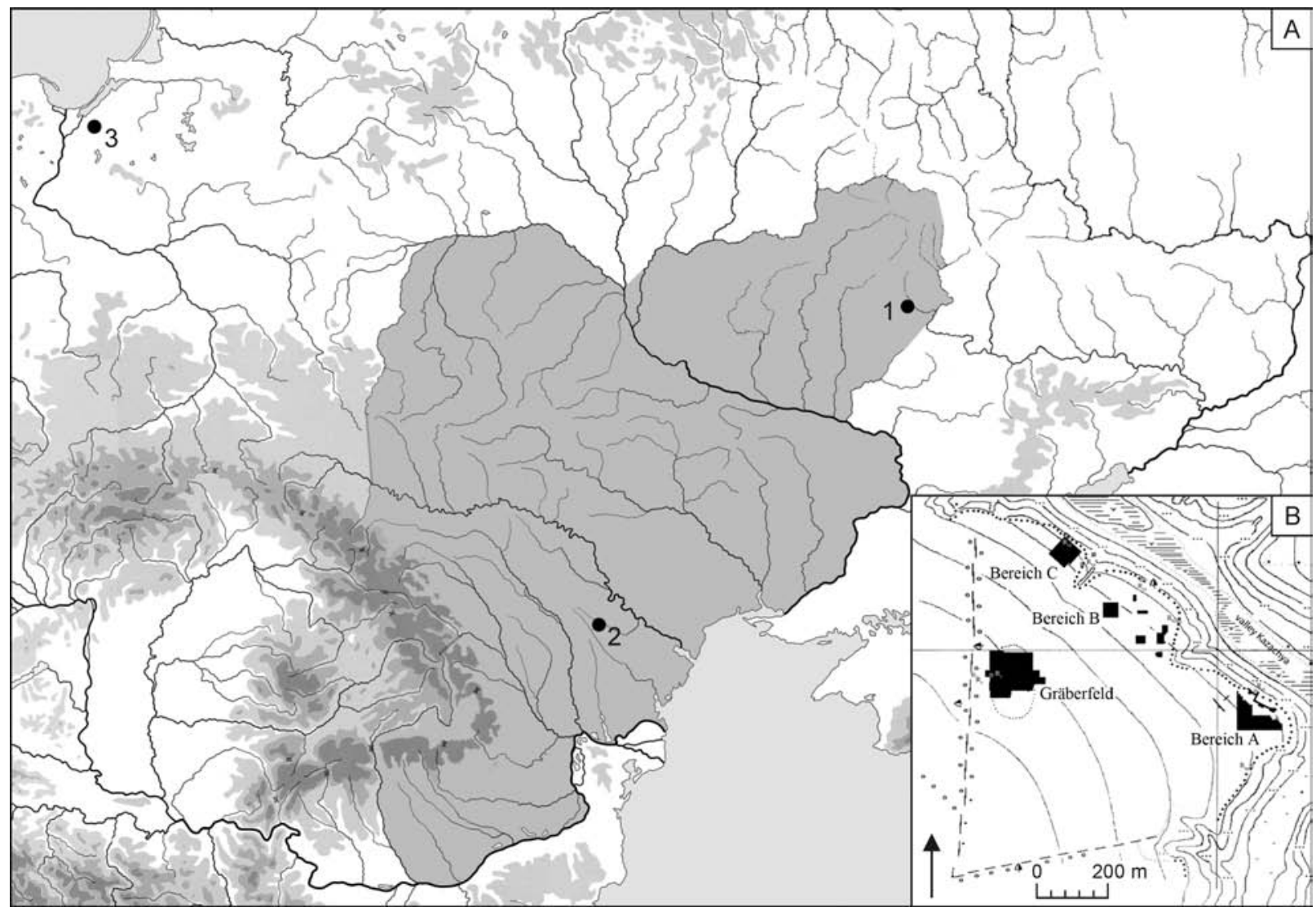

Abb. 1. A - Glasschalen vom Typ Weklice im Barbaricum; B - Plan des archäologischen Komplexes Vojtenki 1. Legende: Grau - Gebiet der Sântana de Mureş-Černjachov-Kultur; 1 - Vojtenki 1; 2 - Dănceni; 3 - Weklice.

\section{GLASSCHALEN}

Obwohl das erste Fragment der Schalen dieses Typs bereits 2009 im Grab 110 des Gräberfeldes gefunden wurde, haben wir erst seit 2015 eine klare Vorstellung über die morphologischen Besonderheiten dieses Glasgefäßtyps bekommen, nachdem ein ganzes Gefäß des Typs im Grab 211 entdeckt wurde.

Dieses Gefäß ist nicht hoch, eine dünnwandige halbkugelförmige Schale mit einem Rundboden, die aus farblosem durchsichtigem Glas mit leicht grüner Färbung gefertigt wurde (Anhang 1, No. 2; Abb. 2: 2). Der Rand der Schale mit rundgeschmolzenem Ende ist uneben und leicht gebogen. Der Boden der Schale ist konkav im Zentrum und trägt Spuren der Benutzung eines Pontils (Nabeleisen). Diese Schale ist durch aufgelegten Faden aus ähnlichem farblosem Glas dekoriert, der eine Komposition in der Art einer wellenförmigen Zickzacklinie bildet. Das Ornament setzt am Boden an und umkreist regelmäßig die Schale.

Die vergleichende Analyse der morphologischen Merkmale ${ }^{3}$ der Fragmente des Glasgefäßes aus Grab 110 (Anhang 1, No. 1; Abb. 2: 1) und der Schale aus Grab 211 (Anhang 1, No. 2; Abb. 2: 2) des Gräberfeldes Vojtenki zeigt Übereinstimmungen beider Erzeugnisse. Vielleicht wurden die beiden Schalen gleichzeitig von einem Handwerker produziert.

Ordnung des Dnepr-Flußsystems (Flüßchen - Mokryj Merčik - Merčik - Merla - Vorskla - Dnepr) im Gebiet der DneprDonec-Wasserscheide (Abb. 1: 1) befindet. Dieser archäologische Komplex wird seit 2004 von der Germanisch-Slawischen archäologischen Expedition der Nationalen W. N. Karazin-Universität Charkow regelmäßig untersucht (Leiter Prof. M. V. Ljubičev). Von 2005 bis 2017 wurden 224 Gräber (113 Brand- und 111 Körperbestattungen freigelegt). Ein Teil dieser Materialien wurde veröffentlicht (Lyubichev 2009, 72-90; 2013, 236-248; Schultze/Lyubichev 2012, 409-463; 2015, 231-255; 2017, 277-294).

3 Nämlich nach der Dicke des Glases, der Art der Randbearbeitung, Methoden der Verzierung und der Komposition des Ornaments. 

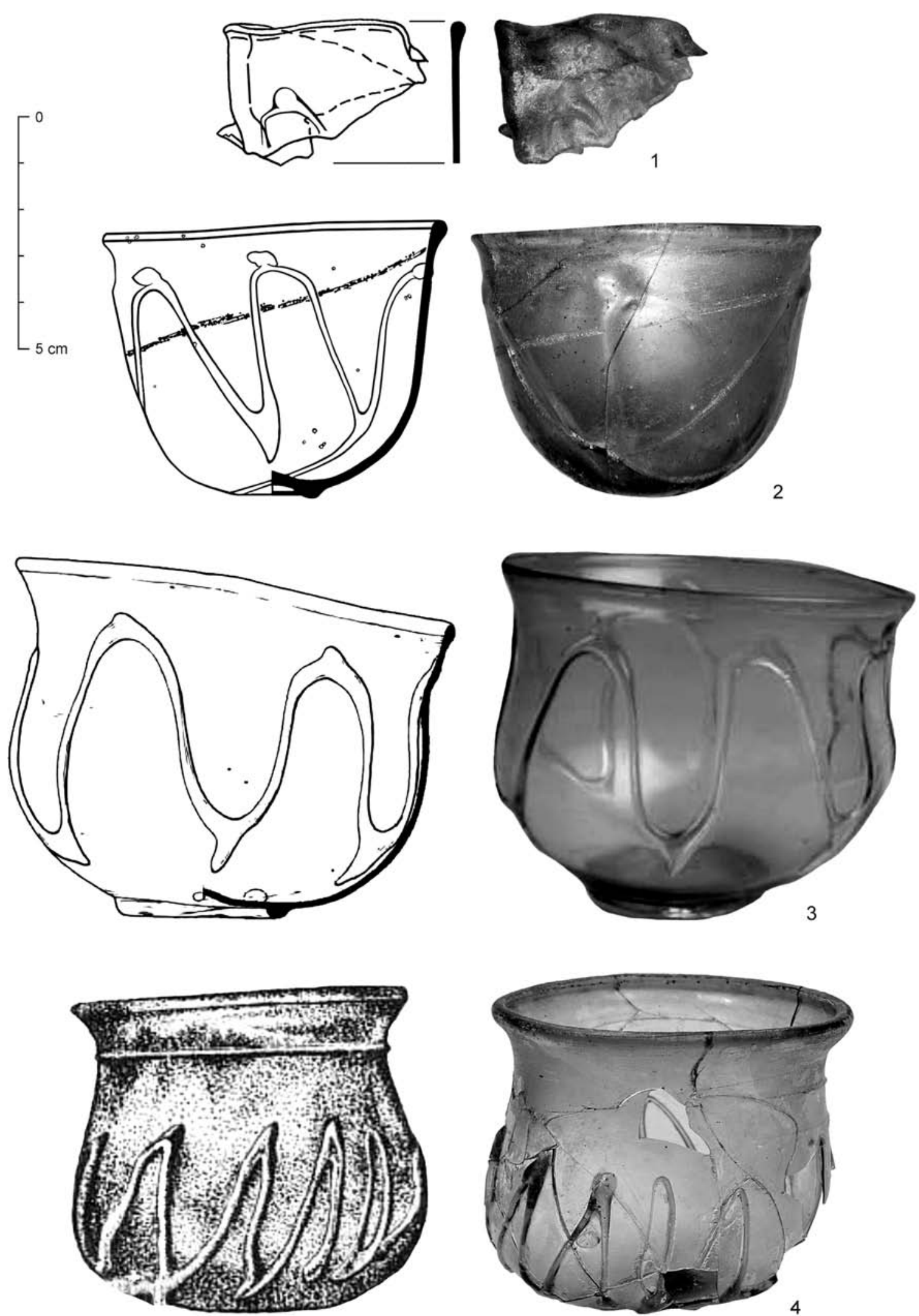

Abb. 2. Glasschale vom Typ Weklice. 1 - Vojtenki 1, Grab 110 (Anhang 1, No. 1); 2 - Vojtenki 1, Grab 211 (Anhang 1, No. 2); 3 - Weklice, Grab 82 (Anhang 1, No. 3; nach Natuniewicz-Sekuta/Okulicz-Kozaryn 2011); 4 - Dănceni, Grab 169 (Anhang 1, No. 4; nach Rafalovich 1986). Foto nach V. Vornic. Ohne Maßstab: 4. 


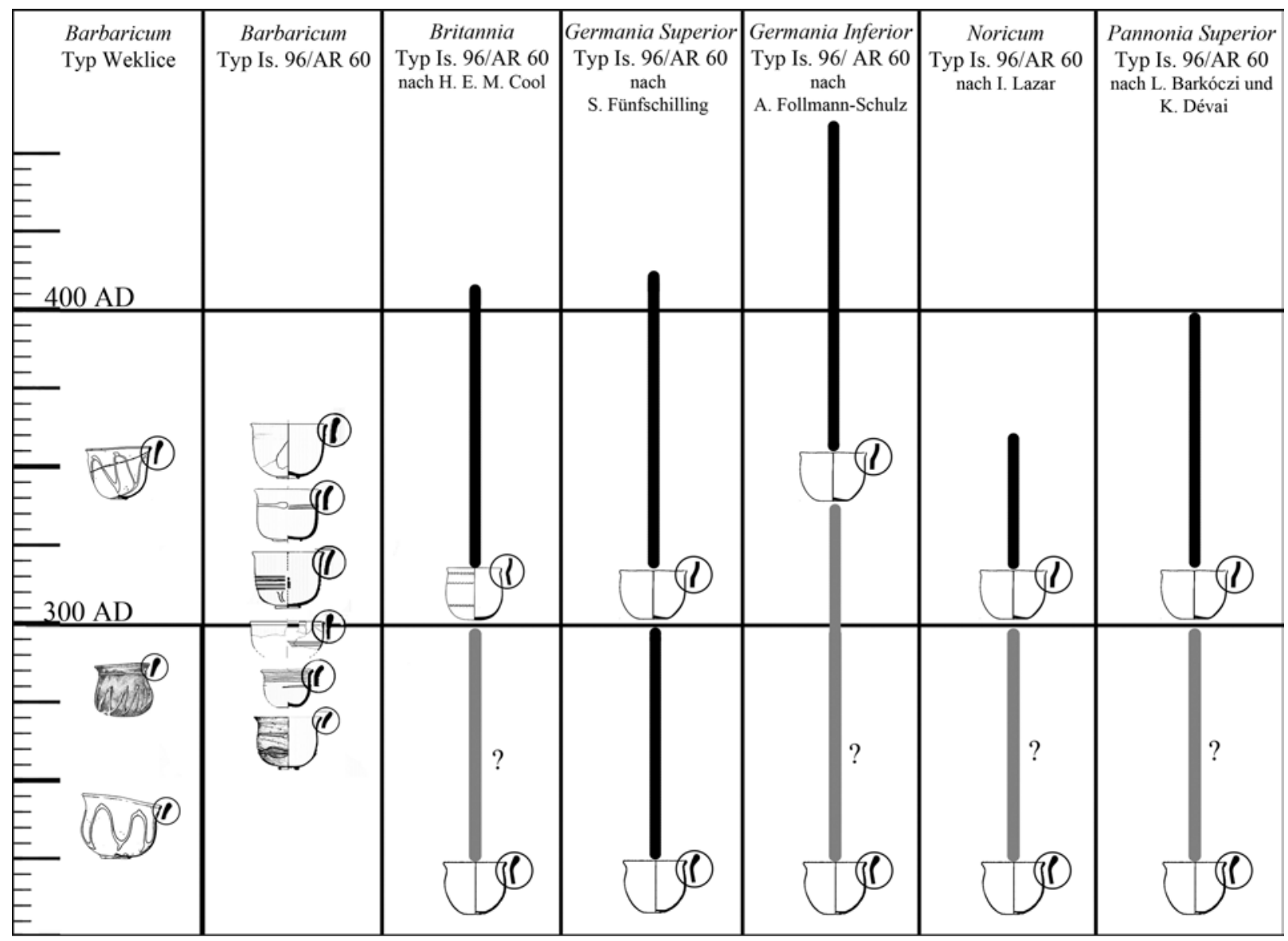

Abb. 3. Chronologie der Verbreitung halbkugeliger Glasschalen mit verschmolzenem und unverarbeitetem/poliertem Rand im Barbaricum und einigen Provinzen des Römischen Reiches. Legende: schwarz - gesicherte Periode des Vorkommens der Schalen; grau - wahrscheinliche Periode des Vorkommens.

Allgemein kann man die Komplexe mit Schalen ähnlicher Form und rundgeschmolzenem Rand in der Sântana de Mureş/Černjachov Kultur in die Stufen C2-C3 datieren ${ }^{4}$ (Abb. 3; Gomolka-Fuchs 1999, 132; Gorokhovski 1988, 42-43; Magomedov 2001, 65; Petrauskas 2003, 265, Abb. 15; 2017, 123-154). Die Gefäße aus den Gräbern 110 und 211 finden keine direkten Übereinstimmungen in den existierenden typologischen Schemata über Glasgeschirr, das aus dem Gebiet der Sântana de Mureş/Černjachov Kultur stammt. Daher muss man diese Gefäße separat betrachten.

Als ihre nächsten Analogien muss man nach unserer Meinung die Gefäße aus Grab 82 des Gräberfeldes Weklice (powiat elbląski, województwo warmińsko-mazurskie, Polen; Abb. 1: 3; Anhang 1, No. 3; Abb. 2: 3) und aus Grab 169 des Gräberfeldes Dănceni (raionul Ialoveni, Republik Moldova; Abb. 1: 2; Anhang 1, No. 4; Abb. 2: 4) anführen.

T. Stawiarska bestimmt die Schale aus Grab 82 des Gräberfeldes Weklice beim Studium der Glasgefäße der Römischen Kaiserzeit auf dem Territorium des modernen Polen als einzelnen Typ, der nach dem Fundort die Bezeichnung Typ „Weklice“ erhielt (Stawiarska 1999, 135). Als Analogien dieser Schale wertet sie nach der Form die Glasgefäße aus Rudka, Černjachov und Malbork-Wielbark, während es ein ähnliches Ornament an der Schale aus Grab 169 des Gräberfeldes Dănceni gibt (Abb. 4: 5, 7-9; Stawiarska 1999, 136, 138).

Während die drei erstgenannten Gefäße nach unserer Meinung sehr entfernte Parallelen zur Schale aus Weklice darstellen (übereinstimmende Merkmale sind nur die Art der Bearbeitung von Rand und Ringboden), gehört das zuletzt genannte Gefäß ohne Zweifel zu den nächsten Analogien. T. Stawiarska datiert die Schale in die Stufe C 2 (Stawiarska 1999, 135), obwohl das Grab eine breitere Datierung in

4 Die Chronologie der „barbarischen“ Komplexe in der Publikation ist mit den zentraleuropäischen Systemen der relativen Chronologie nach den Arbeiten von K. Godłowski und J. Tejral verbunden (Godłowski 1970; Tejral 1986). 


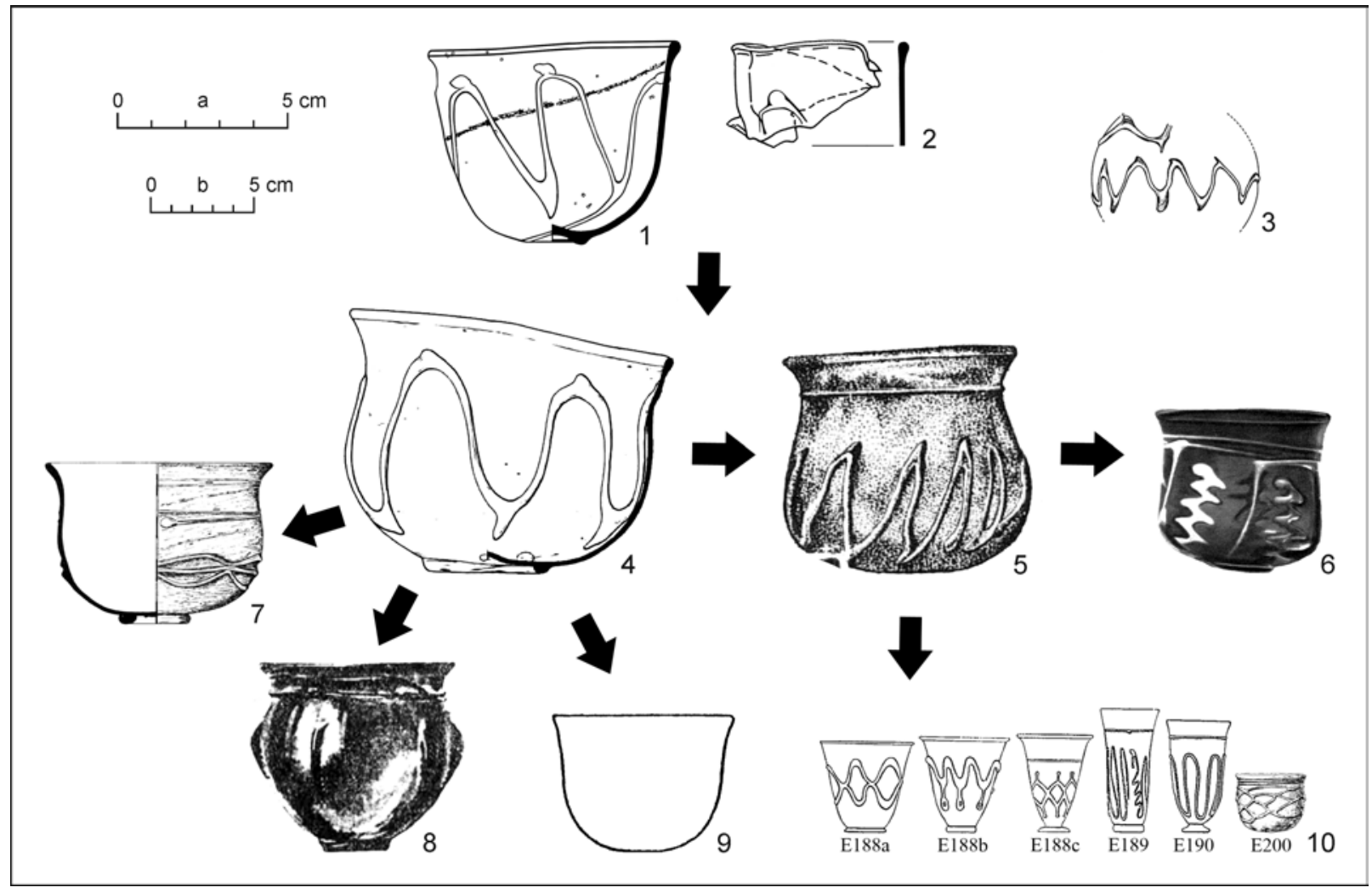

Abb. 4 Glasschalen vom Typ Weklice und ihre Analogien. 1 - Vojtenki 1, Grab 110; 2 - Vojtenki 1, Grab 211; 3 - Marea; 4 - Weklice, Grab 82; 5 - Dănceni, Grab 169; 6 - Nižnij Strutin; 7 - Rudka; 8 - Černjachov, Grab 88; 9 - Malbork-Wielbark, Grab 1180; 10 - Glasschalen des Typs Eggers 200 und Becher der Typen Eggers 188-190. 3 - nach Kucharczyk 2007; 4 - nach Natuniewicz-Sekuła/Okulicz-Kozaryn 2011; 5 - nach Rafalovich 1986; 6 - nach Kropotkin 1970; 7, 8 - nach Rau 1972; 9 - nach Kleemann 2017. 5-8, 9 - Analogien nach T. Stawiarska; 5, 6, 10 - Analogien nach M. B. Shchukin und T. A. Shcherbakova. Maßstab: a - 1, 2, 4; b-6-8; ohne Maßstab - 3, 5, 9, 10 .

die Stufen C1b-C2 (Natuniewicz-Sekuła/Kontny 2010, 141, 142) oder C1b-C2/C2a hat (Natuniewicz-Sekuła/ Okulicz-Kozaryn 2011, 131, 132).

M. B. Ščukin und T. A. Ščerbakova, die Autoren der Chronologie des Gräberfeld Dănceni, erwähnen keine genaue Analogie zur Schale aus dem Grab 169. Als nach Form und Art der Verzierung sehr ähnlich nennen sie ein Gefäß aus dem Hügelgrab des 4. Jhs. bei Nižnij Strutin’ (Ivano-Frankovsk Gebiet, Ukraine; Abb. 4: 6). Jedoch stellt dieser Becher nach ihrer Meinung eine sehr entfernte Analogie dar, da er aus rot-violettem Glas gefertigt und mit Faden aus gelb-blauem Glas dekoriert wurde (Shchukin/Shcherbakova 1986, 192, 193; Parameter und Beschreibung s. Kropotkin 1970, 29, Abb. 78: 3). Insgesamt unterstützen wir diese Zweifeln der Gelehrten und nehmen sogar an, dass nach den wesentlichen morphologischen Unterschieden zwischen den Gefäßen aus Dănceni und Nižnij Strutin` diese auch nicht als entfernte Analogien zu betrachten sind. Im Übrigen werten M. B. Shchukin und T. A. Shcherbakova Glasschalen des Typs Eggers 200 und Becher der Typen Eggers 188-190 als Gefäße mit ähnlicher Komposition und ähnlicher Art des Auftragens der Verzierung auf die Gefäßoberfläche (Abb. 4: 10). Auf dieser Basis datieren sie die Schale aus Dănceni an den Übergang der Stufen C1 und C2 (Shchukin/Shcherbakova 1986, 192, 193). Der Komplex selbst gehört zur Phase Ib der relativen Chronologie des Gräberfeldes, die mit der Stufe C1b synchron ist (Shchukin/Shcherbakova 1986, 210, Abb. 10).

Richten wir jedoch die Aufmerksamkeit auf bestimmte morphologische Unterschiede zwischen den Schalen aus Vojtenki (Anhang 1, No. 1-2; Abb. 2: 1, 2) und den Gefäßen aus Weklice und Dănceni (Anhang 1, No. 3-4; Abb. 2: 3, 4). Erstere haben eine viel geringere Größe, es fehlen bestimmte Konstruktionsteile (wie der Ringbogen bei der Schale aus Weklice) und Dekorelemente (wie der horizontal aufgelegte Faden auf der Schale aus Dănceni). Die Schale aus Grab 211 des Gräberfeldes Vojtenki (Anhang 1, No. 2; Abb. 2: 2) hat zudem eine interessante Besonderheit, die dieses Gefäß von denen aus Weklice 
und Dănceni unterscheidet. Der untere Teil des Gefäßes wurde nach dem Dekorieren durch Wälzen (marvering) $)^{5}$ bearbeitet, wodurch das Ornament deutlich zusammengedrückt wurde. Diese Umstände zeigen, dass die Gefäße von Typ Weklice kaum einen gemeinsamen Ursprung hatten. Trotz dieser Unterschiede gehören diese Schalen jedoch aufgrund der ähnlichen Form, dem gleichen Verfahren bei der Bearbeitung des Randes sowie einer ähnlichen Ornamentkomposition zu einem Typ. Die Funde dieses Typs umfassen im Barbaricum bisher nur drei Komplexe, daher müssen wir weitere Analogien für ihre chronologische Bestimmung suchen.

Einige Fachleute (Rumyantseva/Lyubichev/Trifonov 2018, 187, 188; Stawiarska 2014, 98) verbinden die Herstellung der durch die „heiße Methode ${ }^{\prime \prime 6}$ dekorierten Glasgefäße mit provinzial-römischer Technologie der Glasbearbeitung. Deshalb muss man die Analogien zur Glasschale vom Typ Weklice in der römischen Umwelt suchen.

Natürlich haben die halbkugelige Form und der rundgeschmolzene Rand dieser Gefäße Parallelen unter den Glasgefäßen, die auf dem Territorium des Römischen Reiches hergestellt wurden. Nach Meinung von C. Isings, existieren Schalen ähnlicher Form mit nach außen gebogenem Rand und oft unverarbeitetem Ende des Randes (Typ Isings 96), dekoriert durch Schliff, aufliegende Glasnuppen, Falten, in der Form geblasenem Ornament oder ohne Ornament auf dem Territorium des Reiches während des 3.-4. Jhs. (Isings 1957, 113-115, 131-133). Nach B. Rütti, erscheinen hier solche Gefäße (Typ AR 60 nach der Typologie von Rütti) in der Mitte des 2. Jhs. und existieren bis zum Anfang des 5. Jhs. (Rütti 1991, 330-333). Wir sehen, dass die halbkugelige Form der Erzeugnisse sehr lange unverändert blieb. Dieser Umstand bietet keine Möglichkeit, eine sehr detaillierte Chronologie der Evolution dieser Gefäße zu erstellen. Man muss jedoch eine interessante Beobachtung von S. Fünfschilling in Betracht ziehen, die im Zuge der Forschungen an Glasgefäßen aus Augusta Raurica (Augst, Basel-Landschaft, Schweiz) gemacht wurde. Wie die Archäologin bemerkt, ist die Art der Bearbeitung des Randendes eine wichtige technologische Besonderheit, die Einfluss auf die Chronologie dieser Erzeugnisse hat. Die Schalen, Tassen und Teller mit rundgeschmolzenem Ende des Randes erlangten in der Zeit vom 1.-3. Jh. weite Verbreitung. Später im 4. Jh. wurden sie durch Gefäße mit unverarbeitetem/polierten Randende verdrängt. Eine etwas andere Situation gibt es bei den Bechern, wo eine „Renaissance“ der Erzeugnisse mit rundgeschmolzenem Ende des Randes nach ihrem Verschwinden am Ende des 3. Jhs. in der zweite Hälfte des 4.-Anfang 5. Jhs. vor sich ging (Fünfschilling 2015, 54-56).

Eine ähnliche Situation gibt es im modernen ungarischen Teil der römischen Provinz Pannonia, wo sich halbkugelförmige Schalen mit unverarbeitetem/poliertem Ende des Randes sehr spät, in zweite Hälfte des 4. Jhs. verbreiten ${ }^{8}$ (Barkóczi 1988, 56-58, Taf. II: 17-20), in Britannia, wo ähnliche Gefäße und konische Becher unter dem Tafelgeschirr der ersten zwei Drittel des 4. Jhs. überwiegen ${ }^{9}$ (Cool $1995,12,13)$ und im modernen slowenischen Teil von Noricum, wo solche Erzeugnisse vom Ende des 3. bis zum Ende des 4. Jhs. vorkommen ${ }^{10}$ (Abb. 3; Lazar 2003, 82, 83). Halbkugelförmige Schalen mit unverarbeitetem/poliertem Ende des Randes waren vielleicht ein Erzeugnis der spätrömischen Glaswerkstätten in der Umgebung von Hambach (Nordrhein-Westfalen, Deutschland) in der Germania Inferior (Follmann-Schulz 2015, 25, Abb. 2: 5), die von Mitte des 4. Jhs. bis in das zweite Drittel des 5. Jhs. bestanden (Abb. 3; Brüggler 2009). Das alles erlaubt die Vermutung, dass die von S. Fünfschilling für die Gefäße der Römischen Zeit aus Augusta Raurica beschriebene Erscheinung ${ }^{11}$, nicht nur in der

5 Wälzen (marvering) - Wälzen der Halbfabrikate/Erzeugnisse auf einer flachen, ebenen Oberfläche, um sie in die notwendige Form zu bringen oder die Verzierung zu befestigen.

6 Dieser technologische Prozess ist sehr kompliziert und erfordert daher spezialisierte, professionelle Übung und Fähigkeiten.

7 Hier muss man unterstreichen, dass die Archäologin das Bild der Evolution von Glasgeschirrformen der Römischen Kaiserzeit für das Territorium der modernen nordwestlichen Schweiz entwirft, mit Hinweis darauf, dass sich dieser Prozess in den übrigen Regionen des Reiches etwas anders vollzogen haben kann.

8 Obwohl sie nach Meinung von K. Dévai (2016, 260; 2017, 224, 226). besonders für die Gräber der ersten zwei Drittel des 4 . Jhs. dieser Region charakteristisch sind.

9 Die „Renaissance“ des Tafelgeschirrs mit rundgeschmolzenem Ende des Randes in dieser Region datiert H. Cool nicht früher als Mitte des 4. Jhs. und verbindet ihr Erscheinen mit einer örtlichen Herstellung (Cool 1995, 12, 14).

10 Dazu bemerkt I. Lazar, dass die Gefäße mit rundgeschmolzenen Ende des Randes hier große Verbreitung von Mitte 2.-Mitte 3. Jh. haben (Lazar 2003, 18).

11 Es ist daran zu erinnern, dass nach den Beobachtungen der Archäologin schon in Anfang des 4. Jhs. die Glasgefäße mit unverarbeitetem/poliertem Ende des Randes eine dominante Position innerhalb des Tafelgeschirrs haben. Erzeugnisse mit rundgeschmolzenem Rand wurden praktisch völlig verdrängt. (Fünfschilling 2015, 54-56). 
Germania Superior existierte, sondern auch in einer Reihe der oben genannten Provinzen des Römischen Reiches (Abb. 3).

Art und Komposition der Verzierung der Gefäße vom Typ Weklice haben Parallelen unter den Glasgefäßen in Marea (Qaryat Shakush, Al Iskandariyah, Ägypten) im römischen Ägypten, die aber in eine spätere Zeit gehören ${ }^{12}$. Kugelförmige Gefäße aus farblosem transluzidem Glas wurden durch aufgelegten dunkelblauen Glasfaden verziert (Abb. 4: 3; Kucharczyk 2007, 70-74).

Diese Argumente zeugen von der provinzial-römischen Herkunft der Schalen vom Typ Weklice. Die Herstellungszeit dieser Schalen kann man auf den Zeitabschnitt zwischen dem zweiten Drittel des 3. Jhs. und dem Ende des 3./Anfang des 4. Jhs begrenzen. Das untere Datum stimmt überein mit dem Erscheinen ähnlicher Formen in geschlossenen Komplexen des Barbaricums, das obere Datum bedeutet das Ende der Herstellung/das Verschwinden der Glasgefäße mit rundgeschmolzenem Rand in einigen begrenzten Provinzen des Reiches.

\section{KONTEXT DER FUNDE}

\section{Grab 110}

Der Komplex befindet sich im Ostteil des Gräberfeldes, in gleichem Maße sowohl vom Zentrum wie auch vom Rand entfernt, wenn man von dem bisher untersuchten Teil der Nekropole ausgeht (Abb. 5). Das Grab besteht aus einer Anhäufung kalzinierter Knochen, Resten von Drehscheibenkeramik und anderen Funden auf 0,25 x 0,3 m Fläche, die in einer Tiefe von 0,28-0,39 m in der Schwarzerdeschicht entdeckt wurde ${ }^{13}$ (Abb. 6; Lyubichev/Myzgin/Varacheva 2010, 40, 41) und die man als Brandbestattung des Typs 1 nach O. V. Petrauskas (2002, 43-45) klassifizieren kann - ein Urnengrab.

In der Grube wurden zwei Urnen festgestellt. Eine der Urnen war ein durch Druck im Erdreich zerstörter Drehscheibentopf (Gefäß 1; Anhang 2, No. 6; Abb. 7: 1), die zweite ein ebenfalls zerdrückter Keramikbecher (Gefäß 2; Anhang 2, No. 7; Abb. 7: 2). Beide Gefäße weisen Spuren von Sekundärbrand auf (Lyubichev/Myzgin/Varacheva 2010, 40, 41). Nach Ansicht von O. V. Petrauskas (2002, 42, 43). dienten in den Brandgräbern des Typs 1 Gefäße als Urne, die sich vermutlich nach der Leichenverbrennung erhalten hatten. Die verbrannten Skelettknochen befanden sich in den aufgestellten Gefäßen, jedoch ist anzumerken, dass die Schädelknochen im Becher niedergelegt waren, während die übrigen Knochen des Skelettes offensichtlich in den Topf eingesammelt worden waren. In einer Tiefe von 0,32 m befanden sich bei Gefäß 2 die hier vorgestellten geschmolzenen Fragmente des Glasbechers (Anhang 1, No. 1; Abb. 2: 1). Zwischen diesen und dem Keramikbecher lagen zwei doppelkonische Keramikspinnwirtel (Anhang 2, No. 1-2; Abb. 7: 5, 6; Typ A121a und A111a nach Magomedov 2015, Abb. 2: 5; 3: 11). In Gefäß 2 fanden sich die Fragmente eines verbrannten Dreilagenkammes aus Bein mit Bronzeniet sowie eine verbrannte Karneolperle (Anhang 2, No. 4, 5; Abb. 7: 3, 4; Typ V: 1 nach Gopkalo 2008, 74, 75; Lyubichev/Myzgin/Varacheva 2010, 40, 41) $)^{14}$.

Durch das Fehlen eng zu datierender Gegenstände im Grab bietet sich keine Möglichkeit, die Zeitstellung des Komplexes festzustellen. Da sich dieses Brandgrab jedoch in unmittelbarer Nähe von Körperbestattungen befindet, die zur zweiten Phase des Gräberfeldes gehören ${ }^{15}$ (Abb. 5; Grab 111, 112, 126) und die M. V. Lyubichev daher mit den Stufen C3-C3/D1 gleichsetzt (Lyubichev/Schultze/Gella 2019, Anhang 4, 5), kann man vermuten, dass auch das Brandgrab 110 in diese Phase gehört.

12 Dieser Fund gehört ins 4. Jh. und ist wahrscheinlich mit glasverarbeitenden Werkstätten in Syrien-Palästina verbunden (Kucharczyk 2007, 70-74).

13 Alle Tiefenangaben gehen von der heutigen Oberfläche aus.

14 Übereinstimmend mit der Annahme von O. V. Petrauskas waren nicht alle Reste der Totenverbrennung im Grab zusammengebracht worden. Kleine Fragmente der verbrannten Knochen und des Inventars blieben am Verbrennungsplatz zurück, während größere Reste ins Grab gelangten (Petrauskas 2002, 58-60). Genau deshalb ist über die Zusammensetzung des Grabinventars von Brandbestattungen sehr vorsichtig zu urteilen.

15 Phase „C" der relativen Chronologie des Horizontes der „klassischen“ Černjachov-Kultur in der Dnepr-Donec-Waldsteppe (Lyubichev/Schultze/Gella 2019, Anhang 4, 5). 


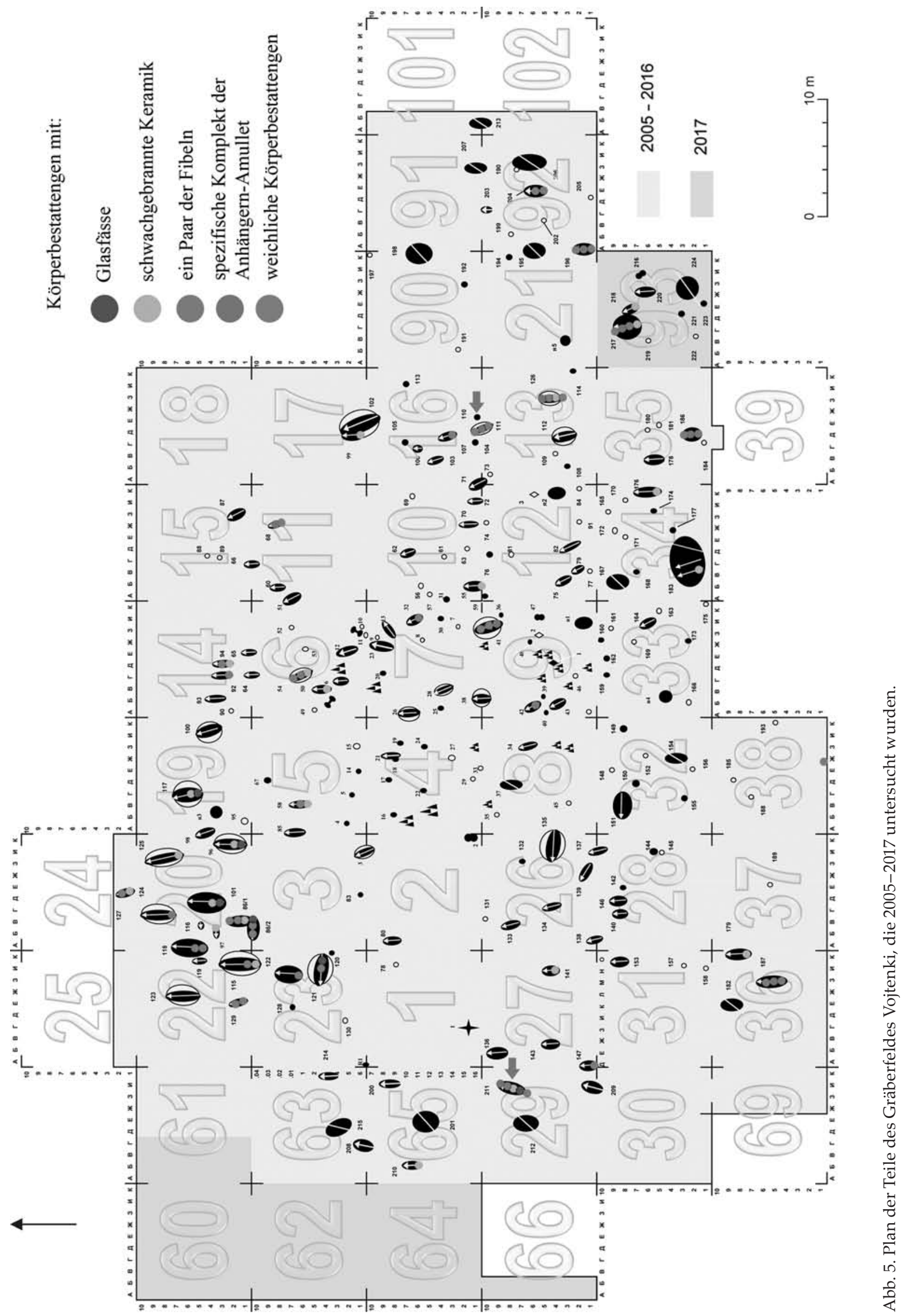




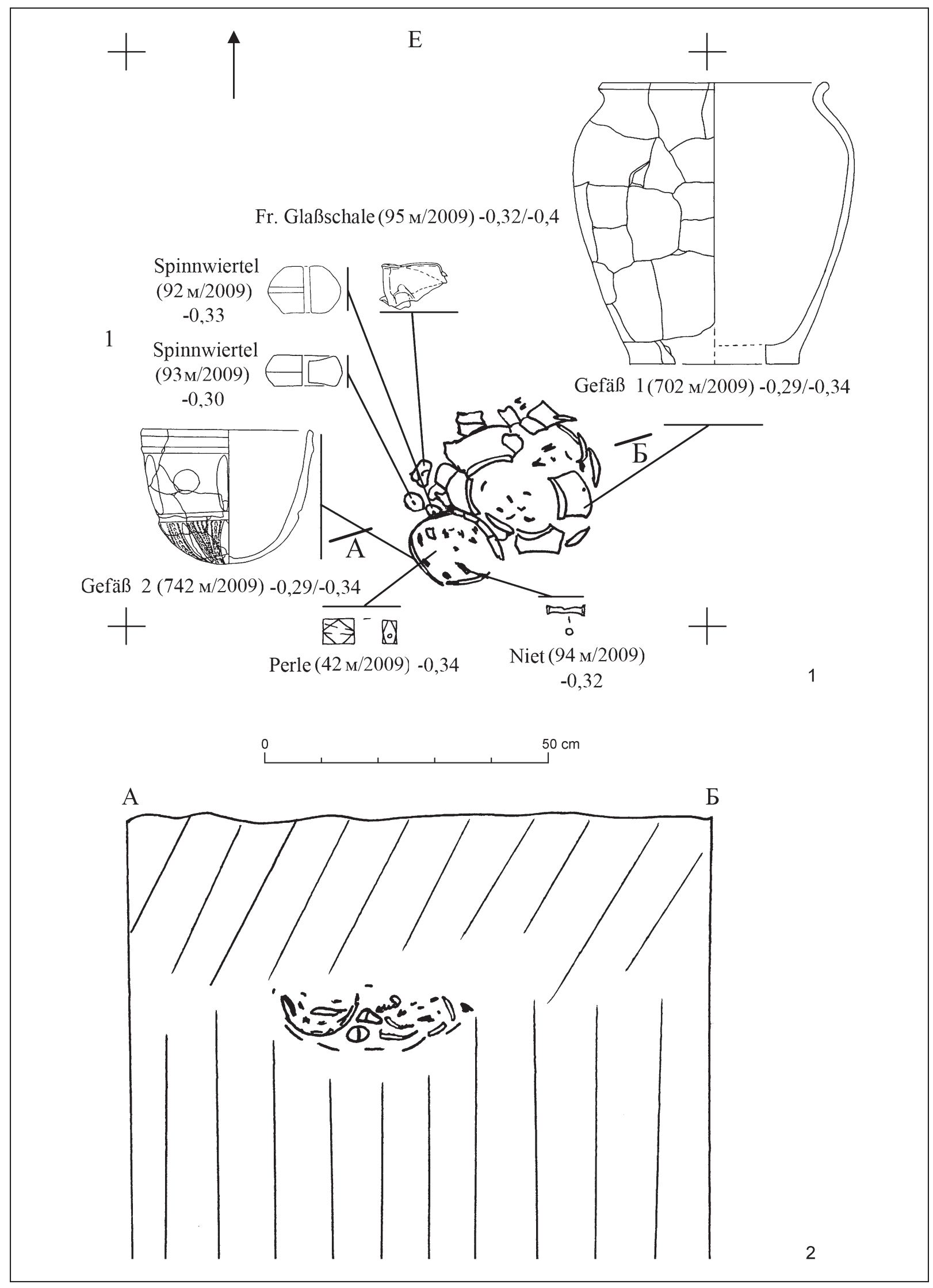

Abb. 6. 1 - Plan; 2 - Profil von Grab 110 des Gräberfeldes Vojtenki 1. 

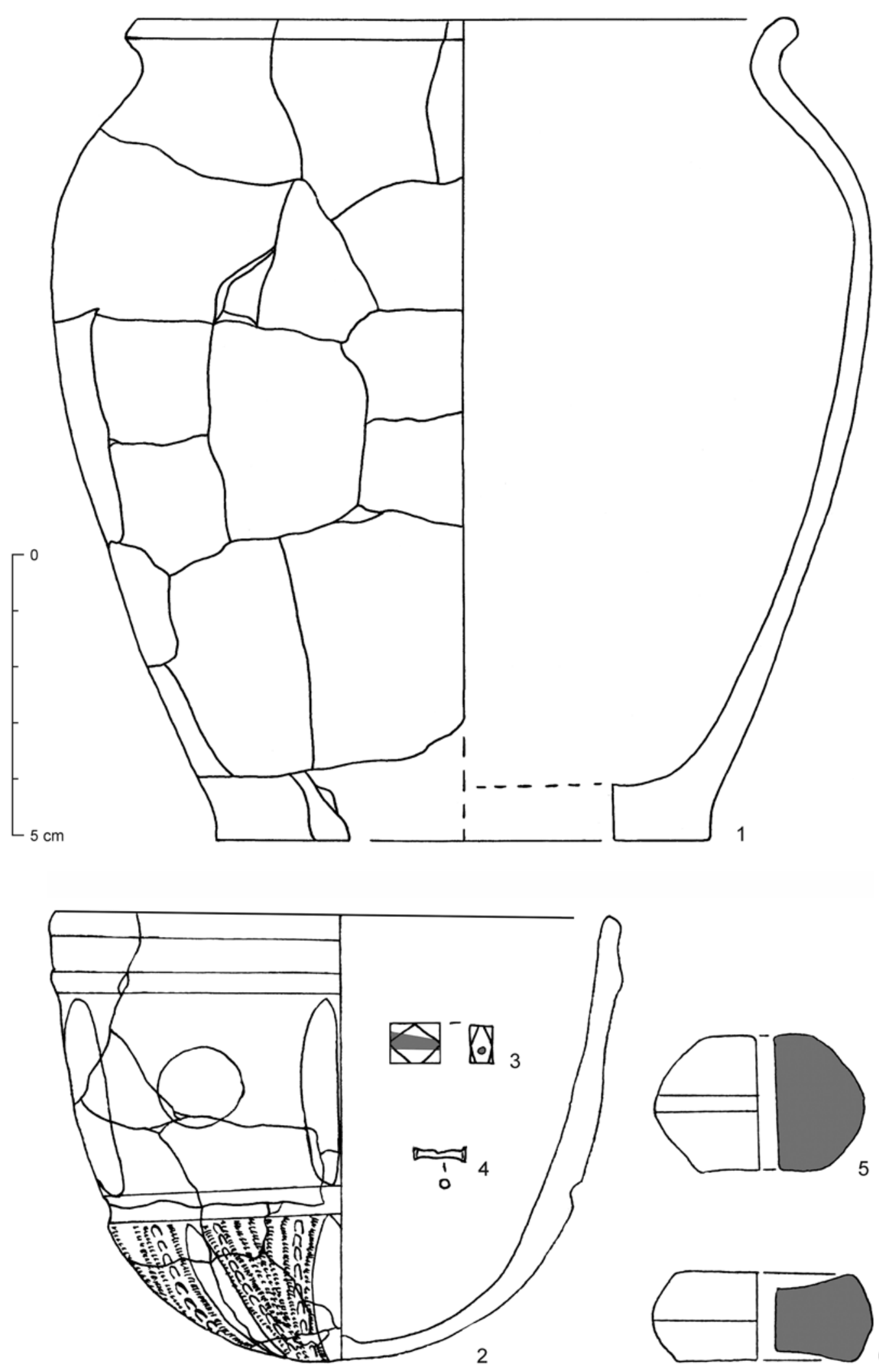

6

Abb. 7. Inventar von Grab 110 des Gräberfeldes Vojtenki 1.1 - scheibengedrehter Topf (Anhang 2, No. 6); 2 - scheibengedrehter Becher (Anhang 2, No. 7); 3 - Karneolperle (Anhang 2, No. 4); 4 - Bronzeniet eines Dreilagenkammes aus Bein (Anhang 2, No. 5); 5 - Keramikspinnwirtel (Anhang 2, No. 2); 6 - Keramikspinnwirtel (Anhang 2, No. 1). 


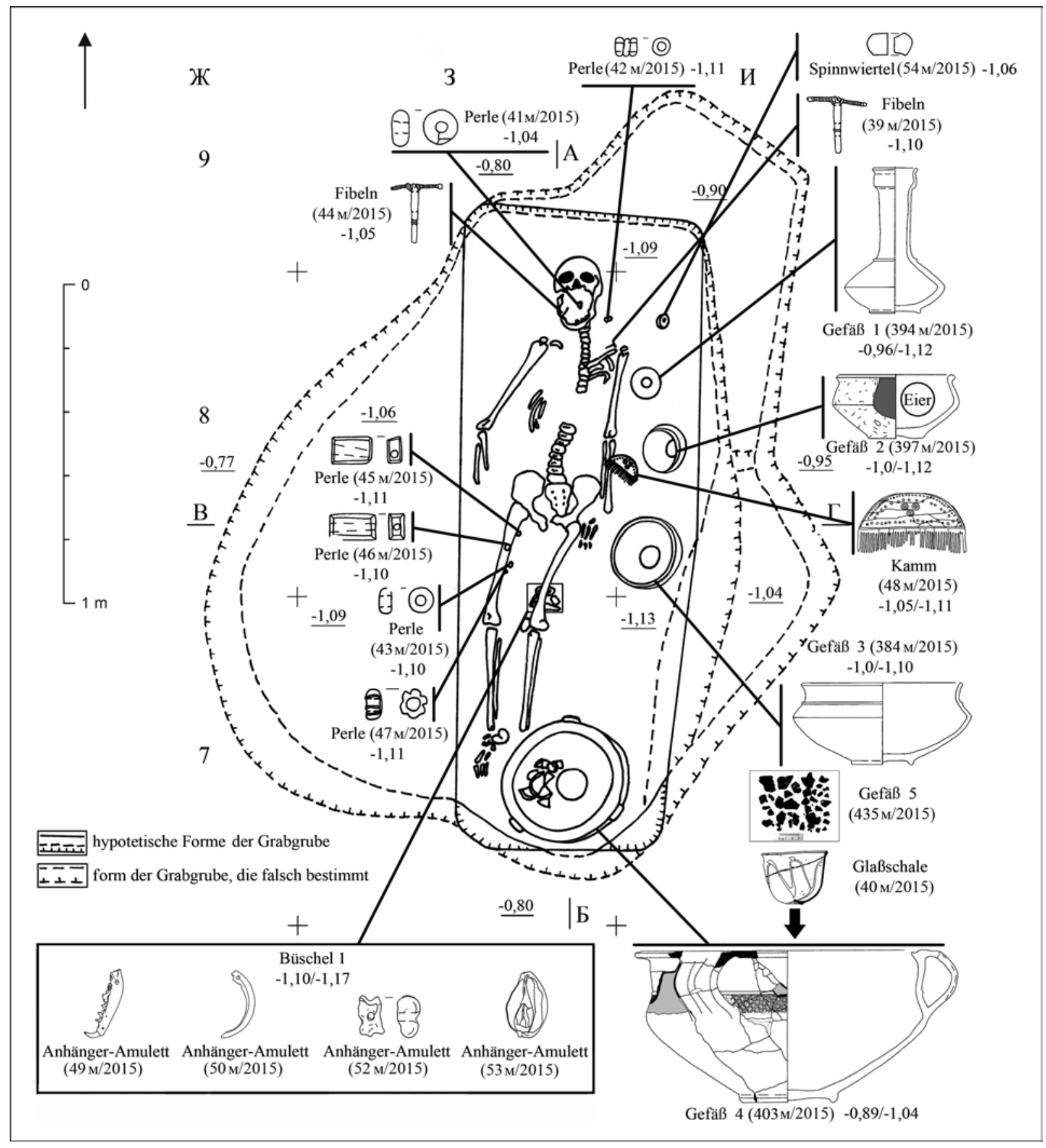

Abb. 8. Plan von Grab 211 des Gräberfeldes Vojtenki 1.

\section{Grab 211}

Das Grab ist im Westteil des Gräberfeldes gelegen, ziemlich nahe an seinem Rand ${ }^{16}$, insofern als auf den in westlich anschließender Richtung untersuchten Sektoren ${ }^{17}$ (29 und 66) der Nekropole nur eine Bestattung entdeckt wurde (Abb. 5).

16 Durch die Arbeiten der Germanisch-Slawischen Archäologischen Expedition gelang es 2018, die Nordwest- und Westgrenze des Gräberfeldes festzustellen (Lyubichev u. a. 2018, 44-52, Abb. 69).

172005 wurde zur Fixierung von Komplexen und Oberflächenfunden eine Einteilung des Gräberfeldes in Sektoren (von 10 x $10 \mathrm{~m}$ ) und Quadrate (von 1 x $1 \mathrm{~m}$ ) mit Nummerierung und Buchstabenbezeichnungen vorgenommen (Lyubichev/Myzgin/ Varacheva 2006, 25-27). 


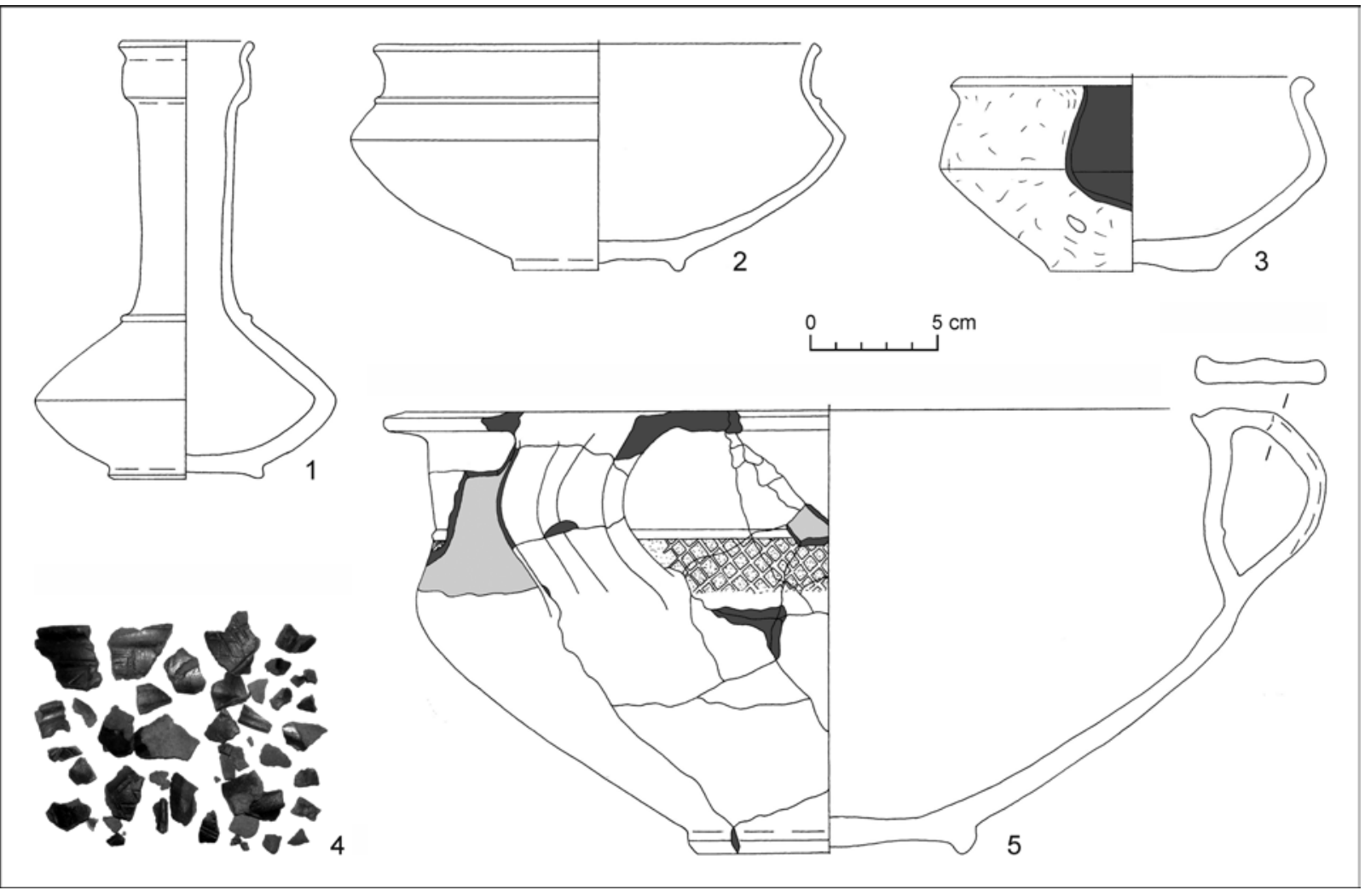

Abb. 9. Inventar von Grab 211 des Gräberfeldes Vojtenki 1. 1 - scheibengedrehte henkellose Kanne (Anhang 2, No. 23); 2 - Schale geschlossenen Typs (Anhang 2, No. 24); 3 - Schale geschlossenen Typs (Anhang 2, No. 25); 4 - scheibengedrehter Becher (Anhang 2, No. 27); 5 - Vase mit drei Henkeln, scheibengedreht (Anhang 2, No. 26).

Das Grab befand sich in einer Tiefe von 1,09-1,16 m, im Übergangshorizont zum anstehenden Boden. Die Verfärbung der Grabgrube zeichnete sich nur schlecht ab, tatsächlich wurden ihre wirklichen Konturen nicht sicher erfasst, deshalb sind die Angaben zu Ausmaßen und Tiefe mit einer gewissen Ungenauigkeit festgelegt worden (Lyubichev u. a. 2016, 54-59).

Das Grab stellt eine Körperbestattung mit einfacher, vermutlich rechteckiger Grube dar; die Bodentiefe betrug 1,09-1,16 $\mathrm{m}$ und die Abmessungen 2,10 x 0,85 $\mathrm{m}^{18}$. Der Boden der Gruben war linsenförmig, deren größte Tiefe im Bereich der Hüfte des Bestatteten festgestellt wurde (Abb. 8; Lyubichev u. a. 2016, 54-59).

Die Tote war eine ältere Frau (55-60 Jahre) ${ }^{19}$, die auf dem Boden der Grube ausgestreckt auf dem Rükken lag und mit dem Kopf nach Norden orientiert war mit einer geringen Abweichung nach Nordosten. Der Oberkiefer, das rechte Schlüsselbein, ein Teil der Rippen, ein Teil der Brustwirbel und die rechte Hand fehlten im Grab. (Abb. 8; Lyubichev u. a. 2016, 54-59) Das ist möglicherweise sowohl mit den Aktivitäten von Tieren wie auch mit der natürlichen Zersetzung feiner und weicher Materialien zu verbinden. Das Phänomen der Zerstörung von Körpergräbern ist auf den Bestattungsplätzen der Černjachov-Kultur zu beobachten, O. V. Petrauskas erklärt ähnliche lokal begrenzte Störungen, verbunden mit dem Fehlen einzelner anatomischer Teile des Skelettes auf die gleiche Weise (Petrauskas 2014, 130, 131).

Zusätzlich zu der hier vorgelegten Glasschale (Anhang 1, No. 2; Abb. 2: 2) wurden im Grab fünf Drehscheibengefäße entdeckt (Anhang 2, No. 23-27; Abb. 9; 1 henkellose Kanne, 2 Schalen geschlossenen Typs, 1 Vase mit drei Henkeln, 1 Becher), zwei zweiteilige Fibeln mit umgeschlagenem Fuß (Anhang 2, No. 8, 9; Abb. 10: 8, 9; Typ B2B nach Gorokhovski 198820, 35), ein Dreilagenkamm aus Bein (Anhang 2, No. 16;

18 Im Zuge der Untersuchung gelang es nicht, die tatsächliche Form der Grube festzustellen, deshalb sind die Abmessungen in Übereinstimmung mit den Außenpunkten der Lage des Inventars angegeben.

19 Mein herzlicher Dank gilt dem Studenten am Institut für Archäologie der Warschauer Universität, V. V. Chuikov, für die anthropologische Bestimmung des Skelettes.

20 Gruppe 16, Untergruppe 2, Serie I, Variante 2 nach A. K. Ambroz (1966, 63, 64, Taf. 11: 9, 10). 

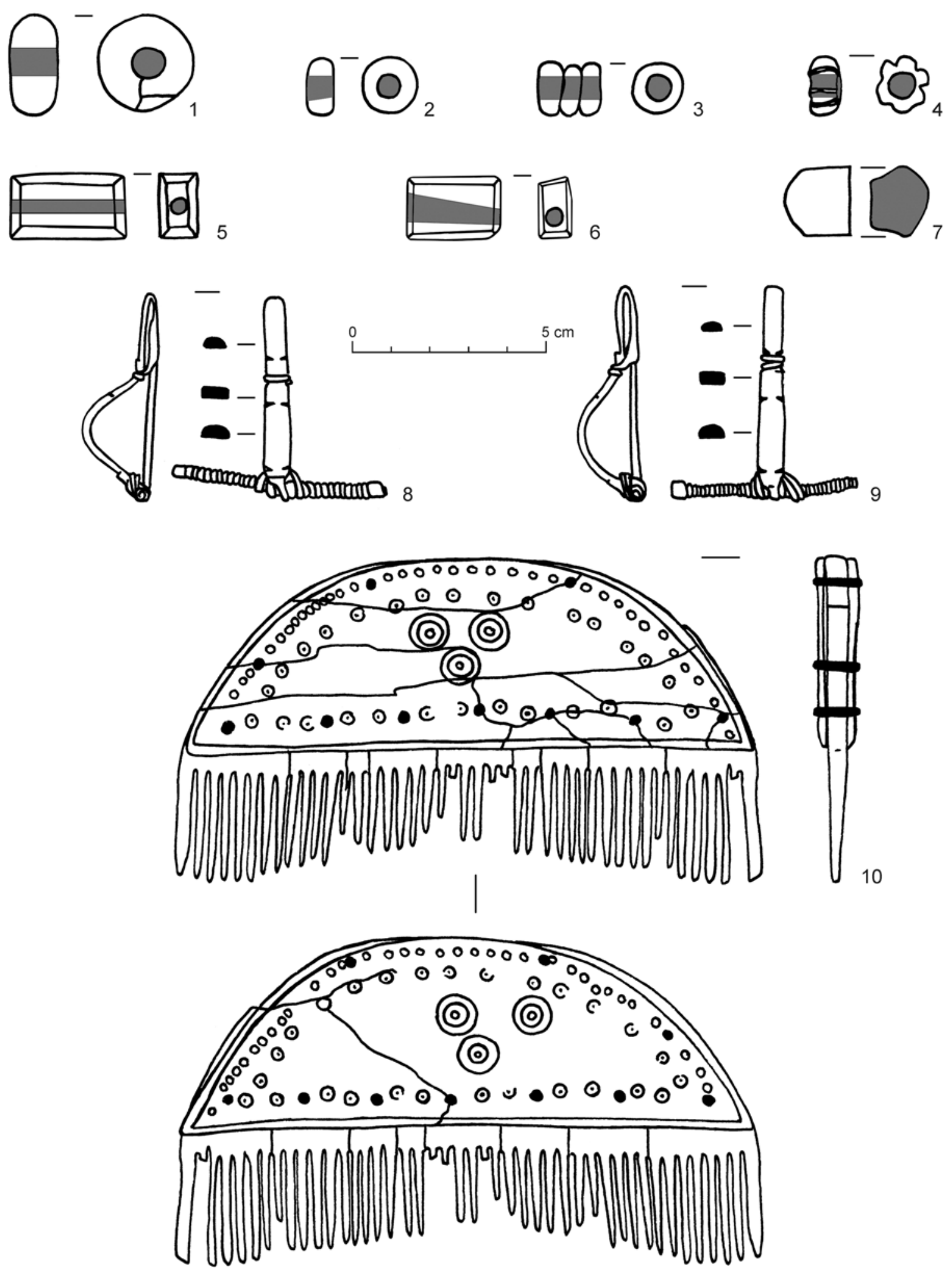

Abb. 10. Inventar von Grab 211 des Gräberfeldes Vojtenki 1. 1 - einfarbige Glasperle (Anhang 2, No. 10); 2 - einfarbige Glasperle (Anhang 2, No. 12); 3 - einfarbige Glasperle (Anhang 2, No. 11); 4 - einfarbige Glasperle (Anhang 2, No. 13); 5 - Karneolperle (Anhang 2, No. 14); 6 - Karneolperle (Anhang 2, No. 15); 7 - Keramikspinnwirtel (Anhang 2, No. 21); 8 - Bronzene zweiteilige Fibel mit umgeschlagenem Fuß (Anhang 2, No. 8); 9 - Bronzene zweiteilige Fibel mit umgeschlagenem Fuß (Anhang 2, 9); 10 - Dreilagenkamm aus Bein (Anhang 2, No. 16). 

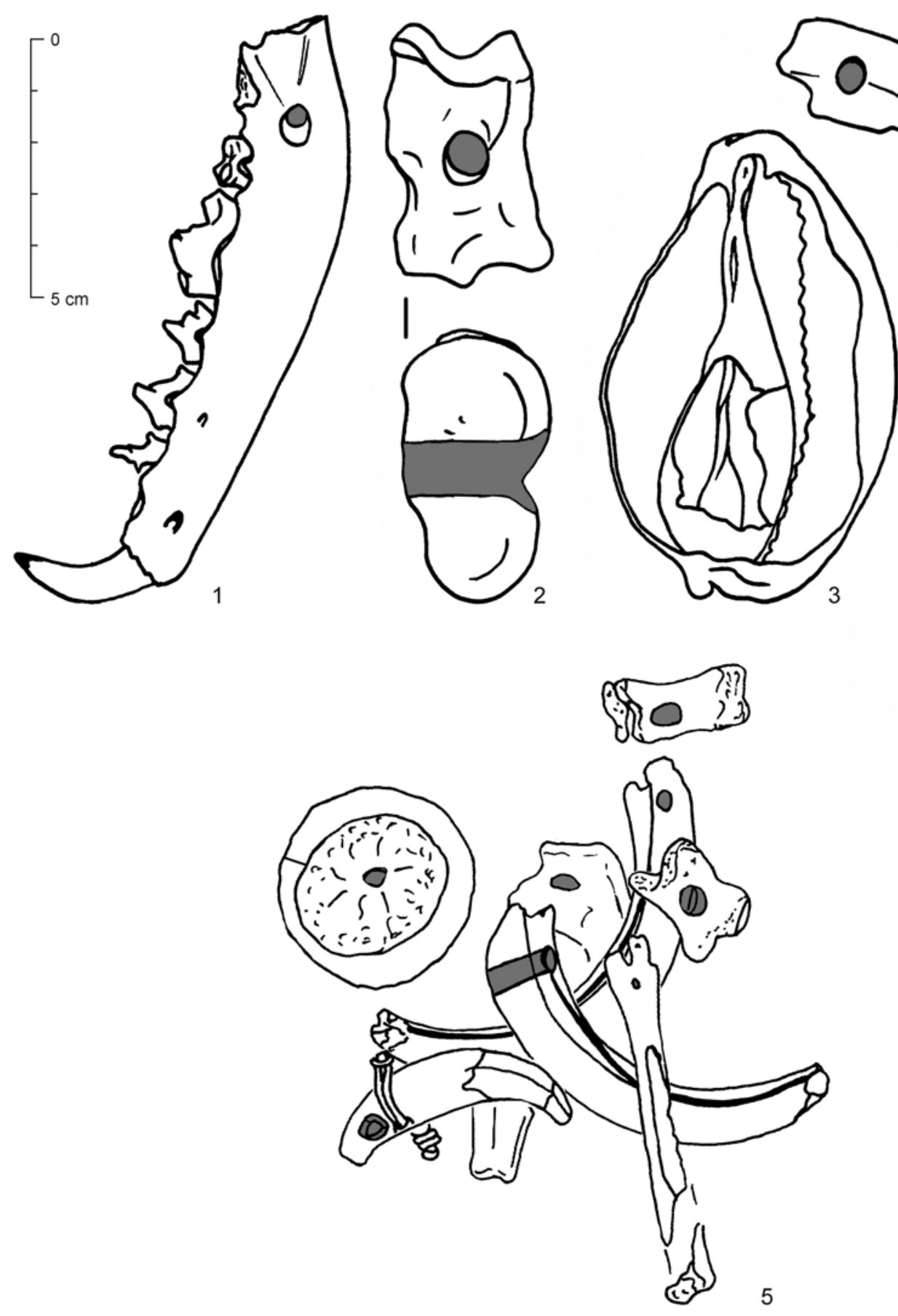

Abb. 11. Garnitur aus der Amulettanhängern aus den Gräbern 211 (1-4) und 68 (5) des Gräberfeldes Vojtenki 1.1 - Amulettanhänger (Anhang 2, No. 20); 2 - Amulettanhänger (Anhang 2, No. 18); 3 - Amulettanhänger (Anhang 2, No. 17); 4 - Amulettanhänger (Anhang 2, No. 19). Ohne Maßstab: 5.

Abb. 10: 10; Typ IB1a nach Nikitina 196921, 147-160), vier einfarbige Glasperlen (Anhang 2, No. 10-13; Abb. 10: 1-4; Untergruppe II: 26; XIII: 1; II: 1, 2; XIV: 11 nach Gopkalo 2008, 16, 21, 22, 32, 35) und zwei Karneolperlen (Anhang 2, No. 14-15; Abb. 10: 5, 6; Typ V: 3 nach Gopkalo 2008, 75, 76), vier Amulettanhänger (s. unten) und ein Spinnwirtel aus Keramik (Anhang 2, No. 21; Abb. 10: 7; Typ C21 nach Magomedov 2015, Abb. 10: 9, 10; Abmessungen und vollständige Beschreibung des Inventars s. Anhang 2).

${ }^{21}$ Klasse I, Serie 2, Variante B nach R. G. Shishkin (1999, 44, 45, Abb. 3-5). 
Tab. 1. Garnitur aus der Amulettanhängern aus den Gräbern des Gräberfeldes Vojtenki 1.

\begin{tabular}{|c|c|c|c|c|c|c|c|c|c|c|c|c|c|}
\hline \multicolumn{13}{|c|}{ Anhänger } & \multirow[b]{2}{*}{ Literatur } \\
\hline 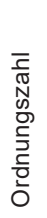 & $\frac{0}{\frac{\pi}{0}}$ & 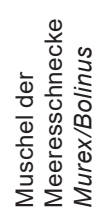 & 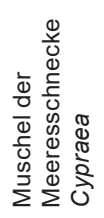 & 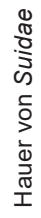 & 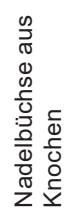 & 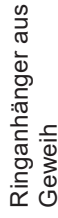 & 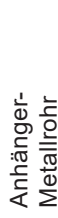 & 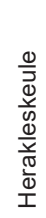 & 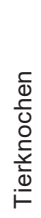 & 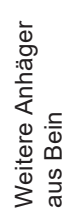 & 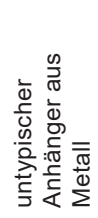 & $\begin{array}{l}\frac{1}{0} \\
\frac{0}{\pi} \\
\frac{\omega}{\sqrt{0}} \\
3\end{array}$ & \\
\hline \multicolumn{14}{|c|}{ Vojtenki 1} \\
\hline 1 & 215 & 2 & 1 & - & - & - & 1 & 2 & - & - & 2 & - & \\
\hline 2 & 41 & 1 & 1 & - & - & - & 1 & - & - & - & - & - & \\
\hline 3 & 111 & 1 & 1 & - & 1 & 1 & - & - & - & - & - & - & \\
\hline 4 & 126 & 2 & - & - & - & - & - & - & 1 & - & - & - & \\
\hline 5 & 217 & 2 & - & - & 1 & - & - & - & - & - & - & - & \\
\hline 6 & 112 & 1 & - & - & 1 & - & - & - & - & - & - & - & \\
\hline 7 & 196 & 1 & - & - & - & 1 & - & - & - & - & - & - & \\
\hline 8 & 186 & 1 & - & - & - & - & - & - & - & - & 1 & 1 & \\
\hline 9 & 187 & 1 & - & - & - & - & - & - & - & - & - & - & \\
\hline 10 & 68 & - & 1 & 3 & - & - & - & - & 4 & 2 & - & - & \\
\hline 11 & 211 & - & 1 & 1 & - & - & - & - & 2 & - & - & - & \\
\hline \multicolumn{14}{|c|}{ Sumy-Sad } \\
\hline 12 & 5 & 1 & 1 & - & 2 & - & - & - & 1 & - & - & - & Nekrasova 1985,76, 77, Abb. 4 \\
\hline \multicolumn{14}{|c|}{ Kompanijcy } \\
\hline 13 & 162 & 1 & 1 & - & - & - & - & - & - & - & - & - & Nekrasova 2006, 114, Abb. 78 \\
\hline \multicolumn{14}{|c|}{ Žovnin/Pristan' } \\
\hline 14 & 25 & 1 & - & - & - & - & - & 2 & - & - & - & - & Rutkovskaya 1979, 324, Abb. 8: 5-7 \\
\hline \multicolumn{14}{|c|}{ Uspenka } \\
\hline 15 & 1647 & 1 & - & - & - & - & - & - & - & - & - & - & Nekrasova 2006, 104, Abb. 52 \\
\hline
\end{tabular}

Die beiden paarigen Bronzefibeln lagen im Bereich der Schultern, zwei Glasperlen ungefähr bei den Halswirbeln, zwei weitere Glas- und zwei Karneolperlen neben dem rechten Oberschenkelknochen der Toten. Eine derartige Lage von Schmuck ist gleichfalls als Resultat von Tieraktivitäten zu betrachten. Östlich des Schädels war in nicht allzu großer Entfernung der Keramikspinnwirtel platziert worden. Oberhalb der linken Speiche war der Kamm aus Bein niedergelegt, aber beim linken Kniegelenk befanden sich vier Amulettanhänger. Östlich des Toten waren vier Drehscheibengefäße verteilt, in einem von diesen (der Dreihenkelvase), das auf dem Fuß der Toten platziert war, befanden sich der Glas- und der Keramikbecher. In einer Schale, die unweit vom Ellenbogengelenk aufgestellt war, wurden Eierschalen entdeckt. (Abb. 8; Lyubichev u. a. 2016, 54-59).

Der im Grab vereinigte Gefäßsatz war nicht nur für die Aufbewahrung von Speisen für den Toten, sondern auch für Getränke bestimmt, wovon seine Zusammensetzung zeugt. Man muss hinzufügen, dass diese Kategorie des Grabinventars eine äußerst interessante Zusammenstellung von Elementen eines reichen rituellen Trinkservices und Küchengeschirrs mit Spuren längerer Nutzung und vermutlich auch Reparaturen darstellt. In Grab 211 bestand dieser Satz aus Drehscheibenkeramik - Schalen, dreihenkliger Vase, henkelloser Kanne und Becher - sowie der Glasschale (Anhang 2, No. 23-27; Abb. 9). Ein derartiges Trinkservice kann man als ,"vollständig“ betrachten, d. h. es umfasst einen Satz aus einer Vase/ schalenartigen Vase bzw. einem dreihenkligem Gefäß, einer Kanne und einem Becher/Tasse (Reida/Heiko/Sapiehin 2015, 32), oder es beinhaltet Töpfe, Schalen offenen und geschlossenen Typs, Vase, dreihenklige Vase, Kannen und Becher (manchmal aus Glas; Lyubichev/Schultze 2018, 122, Abb. 3). Anzumerken ist, dass die Dreihenkelvase und der Becher, die im Grab gefunden wurden, schwach und schlecht gebrannt waren (Anhang 2, No. 26, 27; Abb. 9: 4, 5). Eine der Schalen weist ebenfalls eine interessante Besonderheit auf (Anhang 2, No. 24; Abb. 9: 2). Die Oberfläche des fertigen Gefäßes wurde nochmals mit einer Schicht Ton überdeckt, die vermutlich davon zeugt, dass das Gefäß im Zuge der Benutzung bestimmte Funktionseigenschaften einbüßte und repariert wurde. 
Von besonderem Interesse sind die paarigen zweiteiligen Bronzefibeln mit umgeschlagenem Fuß (Anhang 2, No. 8, 9; Abb. 10: 8, 9). Die Details der Fibeln und ihre Parameter lassen die Vermutung zu, dass diese Gewandschließen identisch sind und nahezu gleichzeitig hergestellt wurden, vermutlich aus einem Stab, geschmiedet.

Bei Betrachtung der Trachtbestandteile der bestatteten Frau darf die Zusammenstellung eines spezifischen Satzes von Amulettanhängern nicht außer Acht gelassen werden. Zu diesem Satz gehören vor allem Anhänger, die aus der Meeresmuschel Cypraea hergestellt wurden, Eckzähnen vom Suidae (Eber?), Knochen (Astragal) von Bovidae (Schaf/Widder?) und dem Unterkiefer eines Canidae (Haushundes; Anhang 2, No. 17-20; Abb. 11: 1-4; Tab. 1, No. 11). Dieses Satz unterscheidet sich ein wenig von seinen Analogien aus Bestattungen, nicht nur vom Gräberfeld von Vojtenki 1, sondern auch in Gräbern anderer bekannter Bestattungsplätze der Černjachov-Kultur in der Dnepr-Donec-Waldsteppe. (Tab. 1).

Die Datierung des Komplexes ist gut feststellbar durch die bronzenen zweiteiligen Fibeln mit umgeschlagenem Fuß vom Typ B2B nach E. L. Gorokhovski $(1988,35)$, die innerhalb der Černjachov-Kultur weite Verbreitung in der Stufe C3 erlangten (Gorokhovski 1988, 43, 44), und den Dreilagenkamm aus Bein vom Typ IB1a nach G. F. Nikitina (1969, 147-160), der hier in einer Periode, begrenzt auf die Stufen C2-C3 (Gorokhovski 1988, 43, 44) vorkam. Im vorliegenden Fall kann man sich der Meinung von M. V. Lyubichev anschließen, der das Grab der zweiten chronologischen Phase des Gräberfeldes Vojtenki 1 bzw. der Periode $\mathrm{C} 3-\mathrm{C} 3 / \mathrm{D} 1$ zuordnet $^{22}$.

\section{SOZIALER STATUS DER GRÄBER}

\section{Grab 110}

Leider ist das Phänomen der Brandbestattungen in der Černjachov-Kultur insgesamt bisher noch wenig erforscht, deshalb ist eine gezielte Rekonstruktion des Sozialstatus der mit Brandritus Bestatteten noch verfrüht. Man kann jedoch die interessante Beobachtung von O. V. Petrauskas anführen, nach der die Zusammensetzung des Inventars in Körpergräbern mit NS-Orientierung und in Brandgräbern praktisch immer identisch ist (Petrauskas 2002, 40-65). Diese Gesetzmäßigkeit trifft höchstwahrscheinlich auch in unserem Fall zu: römischer Import - d. h. Fragmente einer Glasschale, die hier entdeckt wurden - weisen daraufhin, dass das Grab vermutlich zu einer Person mit hohem Sozialstatus gehörte. Allem Anschein nach kann man nach der spezifischen Zusammensetzung des Inventars - speziell nach den zwei Spinnwirteln - vermuten, dass es sich hier um die Brandbestattung einer Frau handelt (Gopkalo 2011, 5, Taf. 3).

\section{Grab 211}

Wie bereits erwähnt gehörte diese Bestattung einer Frau ${ }^{23}$ und war nach Norden orientiert ${ }^{24}$. Dieser Komplex hebt sich durch eine Reihe von charakteristischen Merkmalen heraus. Zu diesen gehören die paarigen Fibeln (unter den weiblichen Gräbern auf dem Gräberfeld wurden paarige Fibeln in 16 von 23 Frauengräbern entdecktt $t^{25} ; 69,5 \%$ ) und die Garnitur spezifischer Amulettanhänger (50 \% unter den

22 Phase "C“ in der relativen Chronologie des Horizontes der „klassischen“ Černjachov-Kultur in der Dnepr-Donec-Waldsteppe (Lyubichev/Schultze/Gella 2019, Anhang 4, 5).

23 Für die vergleichende und statistische Analyse wurden unter den 11 Körpergräbern des Gräberfeldes Vojtenki 175 ungestörte Körperbestattungen ausgewählt. Die Zusammensetzung des Inventars bei 25 davon, oder 33,3 \% der Körpergräber (Grab 32, $41,42,54,58,68,86 / 1,86 / 2,92,94,107,111,118,121,122,124,126,129,147,186,187,196,204,211$, 217), ist eher typisch für Frauengräber (Gopkalo 2011, 5, Taf. 3; Lyubichev/Myzgin/Varacheva 2007, 29-32, 36-45; 2008, 39-44, 61-64; 2009, 44-52, 60-63; 2010, 35, 36, 39, 40; 2011, 45-55; 2012, 56-58; Lyubichev u. a. 2014, 57-60; 2015, 45, 46, 52-54; 2016, 54-59; 2017, 53-60).

24 Unter den weiblichen Körperbestattungen des Gräberfeldes ist die Mehrzahl nach Norden orientiert (23; 92 \%), während Gräber mit Orientierung nach Westen nur in zwei Fällen erfasst wurden (8\%). Die Körperbestattungen mit WO-Orientierung stellen im Unterschied zu den Körpergräbern mit Orientierung des Kopfes nach Norden und den Brandbestattungen eine ganz andere Bestattungsform der Černjachov-Gräberfelder dar, die nicht nur die Orientierung des Leichnams wiederspiegelt, sondern auch in bedeutendem Maße Einfluss auf das dazugehörige Grabinventar hat (Petrauskas 2009, 189, 190). Deshalb werden diese Gräber hier nicht in einem Kontext mit den Körpergräbern mit nach Norden orientiertem Kopf betrachtet.

25 Gräber 41, 54, 86/1, 86/2, 111, 118, 122, 124, 126, 129, 186, 187, 196, 204, 211, 217 (Lyubichev/Myzgin/Varacheva 2007, 36-43; 2008, 39-44; 2009, 44-48; 2010, 35, 36; 2011, 45-47, 51-55; 2012, 56-58; Lyubichev u. a. 2014, 57-60; 2015, 45, 46, 52-54; 2016, 54-59; 2017, 53-60). 
Frauengräbern mit paarigen Fibeln; Tab. 1). Wie schon früher angemerkt, unterscheidet sich die Zusammensetzung dieser Garnitur in Grab 211 von ihren Analogien aus anderen Körpergräbern mit paarigen Fibeln. Eine relativ ähnliche Garnitur von Amulettanhängern stammt aus Grab 68, wo die Bestattung jedoch ein eher dürftiges Inventar aufweist (Abb. 11: 5). Im Falle von Grab 211 kann man vermuten, dass die Amulettgarnitur nicht als Schmuck oder Bestandteil der Bekleidung diente, sondern ein mit einer bestimmten Funktion (mit Kult?) verbundenes Objekt darstellte. Eine weitere Besonderheit des Grabes bildet das „,vollständige“ Trinkservice. Auf dem Gräberfeld Vojtenki 1 wurden unter den 23 weiblichen Körperbestattungen in neun Fällen (39 \%; Grab 41, 54, 86/1, 111, 124, 126, 204, 211, 217) solche Service entdeckt. Es ist auch wichtig zu erwähnen, dass unter den 23 Körperbestattungen von Frauen mit NS-Orientierung nur in Grab 211 das Service von Gefäßen aus Drehscheibenkeramik durch einen Glasschale komplettiert wird. Alle übrigen Funde von Glasschalen in Gräbern mit NS-Orientierung gehören am ehesten zu Männergräbern oder zu Körpergräbern von Frauen mit Orientierung des Kopfes nach Westen (Lyubichev/Myzgin/Varacheva 2009, 44-48; 2010, 50-57; 2011, 48-50, 63, 64; Lyubichev u. a. 2016, 54-59). Unter anderem zieht die relativ dürftige Zusammensetzung des in den Gräbern gefundenen Geschirrs, die nicht zu einem Service, d. h. zu dem für den Bestatteten zubereiteten Mahl gehören, die Aufmerksamkeit auf sich. Die mittlere Anzahl von Gefäßen, die für die Unterbringung und Verwendung der Lebensmittel in weiblichen Körpergräbern mit NS-Orientierung vorgesehen war, besteht aus sechs, in Bestattungen mit paarigen Fibeln und rituellem Trinkservice aber aus 7,5 Einheiten. In der Zeit wie in Grab 211 besteht dieser Satz nur aus zwei (Lyubichev/Myzgin/Varacheva 2007, 29-32, 36-45; 2008, 39-44, 61-64; 2009, 44-52, 60-63; 2010, 35-36, 39, 40; 2011, 45-55; 2012, 56-58; Lyubichev u. a. 2014, 57-60; 2015, 45, 46, 52-54; 2016, 54-59; 2017, 53-60). Schließlich unterscheidet sich dieses Grab von den anderen auch durch das Fehlen von Fleisch unter den Speisebeigaben, die acht von zehn Frauengräber des Bestattungsplatzes (80\%; Grab 41, 54, 86/1, 111, 124, 126, 204, 217) mit einer ähnlichen Trachtbestandteilen und einer ähnlicher Zusammensetzung bei dem Drehscheibengeschirr des Trinkservices hatten (Lyubichev/ Myzgin/Varacheva 2007, 29-32; 2008, 39-44; 2009, 44-52; 2010, 39, 40; 2011, 45-47, 52-55; Lyubichev u. a. $2015,52-54 ; 2017,53-60)$.

Ausgehend von den aufgezählten Besonderheiten des Inventars innerhalb der Körperbestattungen von Frauen dieses Bestattungsplatzes, mit Orientierung nach Norden, mit paarigen Fibeln und „vollständigem“ Trinkservice, kann man vermuten, dass die Bestattete im Leben eine besondere soziale Stellung innehatte, vielleicht verbunden mit der Durchführung kultischer Praktiken. Diesen besonderen Status, genauer seine hohe Bedeutung für ihre Zeitgenossen, unterstreicht auch ein im Grab entdeckter, ziemlich einmaliger, in der Herstellung komplizierter Gegenstand wahrscheinlich provinzialrömischer Herkunft - die Glasschale.

\section{SCHLUSSFOLGERUNGEN}

Ungeachtet der Feststellung spezieller morphologischer Merkmale kann man die hier vorgelegten Glasschalen dem Typ Weklice zurechnen. Funde derartiger Stücke im Barbaricum sind einzigartig, sie sind höchstwahrscheinlich provinzialrömischer Herkunft. Obgleich die Herstellungszeit ähnlicher Gefäße, soweit die Formentwicklung der Glasgefäße im römischen Umfeld beurteilt werden kann, auf die Zeit zwischen dem 2. Drittel des 3. Jhs. und dem Übergang vom 3. zum 4. Jh. begrenzt ist, zeigt die Datierung der Gräber 110 und 211 des Gräberfeldes Vojtenki 1, dass derartige Gegenstände im Barbaricum ziemlich lange weiter bestanden und in deutlich spätere Komplexe gelangten, mindestens der Stufe C3.

Auf dem Gräberfeld Vojtenki 1 sind diese Schalen nur mit Frauengräbern verbunden, in denen die Bestatteten einen hohen oder besonderen sozialen Status hatten. Dabei finden sich die typengleichen Gegenstände in Grabinventaren mit unterschiedlichen Bestattungssitten. Die gewaltigen Ausmaße der Grabgrube und die Reste einer verbrannten Holzkonstruktion in Grab 169 des Gräberfeldes Dančeni (Rafalovich 1986, 63-65), aber auch die vom Standard abweichende Konstruktion der Grabgrube (der Tote wurde in einem Boot bestattet), silberne Trachtbestandteile und Teile der Waffenausrüstung eines Reiters in Grab 82 des Gräberfeldes Weklice (Natuniewicz-Sekuła/OkuliczKozaryn 2011, 131, 132), weisen darauf hin, dass sich die mit ähnlichen Schalen beigesetzten Personen im Leben ebenfalls durch eine hohe soziale Position auszeichneten. Dies erlaubt die Vermutung, dass diese Glasgefäße selbst als einer der Marker für den besonderen Sozialstatus ihrer Besitzer gedient haben können. 


\section{ANHANG 1}

\section{Glasschalen vom Typ „Weklice“}

\section{Gräberfeld Vojtenki 1}

(Kreis Valki, Bezirk Charkiw, Ukraine; Abb. 2: 1)

1. Grab 110 (Abb. 2: 1)

Erhaltungszustand: Randfragment, verschmolzen.

Form: halbkugelförmige Schale.

Oberfläche angegriffen durch Irisation, zeigt Spuren von Feuereinwirkung.

Glasfarbe: transluzid, farblos mit grünlicher Tönung.

Glasmasse enthält Bläschen als Einschlüsse, nicht größer als $1 \mathrm{~mm}$ Durchmesser.

Rand leicht nach außen geneigt, Randende im Zuge der Fertigstellung des Gefäßes rundgeschmolzen.

Art der Verzierung: Aufgelegte Fäden aus Glas der gleichen Farbe wie die des Gefäßes.

Komposition der Verzierung: Der Glasfaden zeichnet eine Komposition aus einer sinus-/wellenartigen Zickzacklinie, die das Gefäß gleichmäßig umgibt. Unter dem Rand an den Bruchstellen ist der Faden durch einen Tropfen aus ähnlichem Glas verbunden.

Abmessungen (cm): Höhe (erh.) 3,1 ; Glasdicke (Rand/Wandung) 0,25/0,15.

2. Grab 211 (Abb. 2: 2)

Erhaltungszustand: ganzes Gefäß.

Form: halbkugelförmige Schale.

Oberfläche hat eine leicht irisierende Patina, die die Bestimmung der realen Farbe etwas behindert.

Glasfarbe: transluzid, farblos mit grünlicher Tönung.

Glasmasse enthält Bläschen als Einschlüsse, nicht größer als 2 mm Durchmesser.

Rand uneben, leicht nach außen geneigt, Randende rundgeschmolzen.

Boden abgerundet, in der Mitte eine kleine Wölbung. Eine kleine flache Fläche auf der Unterseite gewährt dem Gefäß Stabilität auf horizontaler Oberfläche. Am Boden ist die Nutzung eines Pontils (Nabeleisen) sichtbar.

Art der Verzierung: Aufgelegte Fäden aus Glas der gleichen Farbe wie die des Gefäßes.

Komposition der Verzierung: Der Glasfaden zeichnet eine Komposition aus einer sinus-/wellenartigen Zickzacklinie, die das Gefäß gleichmäßig umgibt. Das Ornament beginnt am Gefäßboden. Unter dem Rand und im bodennahen Teil des Gefäßes ist der Faden an den Bruchstellen durch einen Tropfen aus ähnlichem Glas verbunden. Der Bodenteil des Gefäßes ist nach dem Auftragen des Dekors durch Wälzen (marvering) bearbeitet.

Art der Fertigstellung: Blasen in der Form mit Drehbewegung.

Abmessungen (cm): Höhe 5,8; Randdurchmesser 8; mittlerer Durchmesser 6,9; Bodendurchmesser (Stabilitätsfläche)

2; Glasdicke (Rand/Gefäßmitte/Boden) 0,25-0,3/0,2/0,4.

\section{Gräberfeld Weklice}

(powiat elbląski, gmina Elbląg, województwo warmińsko-mazurskie, Polen; Abb. 1: 3)

3. Grab 82 (Abb. 2: 3)

Erhaltungszustand: ganzes Gefäß.

Form: halbkugelförmige Schale.

Glasfarbe: transluzid, farblos mit gelb-grünlicher Tönung.

Glasmasse enthält Bläschen als Einschlüsse von bis zu 4 mm Durchmesser.

Rand uneben, leicht nach außen geneigt, Randende rundgeschmolzen.

Boden abgerundet, auf Standring, im Zentrum eine Wölbung.

Art der Verzierung: Aufgelegte Fäden aus Glas der gleichen Farbe wie die des Gefäßes.

Komposition der Verzierung: Der Glasfaden zeichnet eine Komposition aus einer sinus-/wellenartigen Zickzacklinie, die das Gefäß gleichmäßig umgibt. Das Ornament beginnt an den Wänden (am Mittelteil) des Gefäßes. Unter dem Rand und im bodennahen Teil des Gefäßes ist der Faden an den Bruchstellen durch einen Tropfen aus ähnlichem Glas verbunden.

Art der Fertigstellung: Blasen in der Form mit Drehbewegung.

Abmessungen (cm): Höhe 8; Randdurchmesser 10; mittlerer Durchmesser 9,3; Bodendurchmesser 3,8; Glasdicke (Rand/Gefäßmitte/Boden) 0,35/0,2/0,2 .

Nachweis: Natuniewicz-Sekuła/Okulicz-Kozaryn 2011, 42, Pl. XXXV: 10; CCXLV: 1.

\section{Gräberfeld Dănceni}

(raionul Ialoveni, Republica Moldova; Abb. 1: 2)

4. Grab 169 (Abb. 2: 4)

Erhaltungszustand: ganzes Gefäß.

Form: halbkugelförmige Schale. 
Glasfarbe: transluzid, farblos mit grünlicher Tönung.

Rand nach außen geneigt, Randende rundgeschmolzen.

Boden abgerundet, im Zentrum eine Wölbung. Eine kleine flache Fläche auf der Unterseite gewährt dem Gefäß Stabilität auf horizontaler Oberfläche.

Art der Verzierung: Aufgelegte Fäden aus Glas der gleichen Farbe wie die des Gefäßes.

Komposition der Verzierung: Der Glasfaden zeichnet eine Komposition aus einer sinus-/wellenartigen Zickzacklinie und einer etwas höher gelegenen horizontalen Linie, die das Gefäß gleichmäßig umgeben. Das Ornament beginnt an den Wänden (am Mittelteil) des Gefäßes.

Abmessungen (cm): nach der Information aus der Publikation lassen sich die Abmessungen des Gefäßes nicht feststellen.

Nachweis: Rafalovich 1986, 64, Taf. XXXI: 10.

\section{ANHANG 2}

\section{Katalog der Funde aus den Gräbern 110 und 211 des Gräberfeldes Vojtenki}

\section{Grab 110}

1. Keramikspinnwirtel (93 m/2009; Abb. 7: 6)

Form: doppelkonisch, mit scharfem Umbruch und konkaver Grundfläche.

Oberfläche: glatt, Farbe braun.

Maße (cm): Höhe 1,5; Durchmesser der Grundfläche 3,2; größter Durchmesser 4; Durchmesser des Loches 0,7.

2. Keramikspinnwirtel (92 m/2009; Abb. 7: 5)

Form: doppelkonisch, mit unscharfem Umbruch und ebener Grundfläche.

Oberfläche: glatt, Farbe - braun.

Maße (cm): Hoch 2,4; Durchmesser der Grundfläche 2,2; Durchmesser des Umbruchs 4; Durchmesser des Loches 0,8.

3. Fragmente der Glasschale (95 m/2009)

Parameter und Beschreibung s. Anhang 1.

4. Karneolperle (42 m/2009; Abb. 7: 3)

Form: mit 14 Kanten, Quader mit geschliffenen Kanten.

Durchlochung: zylindrisch.

Oberfläche: teils verbrannt.

Maße (cm): Länge 0,9; Querschnitt 0,5 x 0,7; Durchmesser des Loches 0,2.

Chronologie: letztes Drittel 3. - erste Hälfte des 4. Jhs. (Gopkalo 2008, 74, 75).

5. Bronzeniet eines Dreilagenkammes aus Bein (94 m/2009; Abb. 7: 4)

Maße (cm): Länge 0,9; Querschnitt 0,2.

6. Scheibengedrehter Topf (Gefäß 1; 702 m/2009; Abb. 7: 1)

Zustand: Rekonstruktion möglich.

Form: mit rundem Bauch, größter Durchmesser im oberen Drittel des Gefäßes.

Oberfläche: rau, Farbe: grau, orange mit Spuren von sekundärem Brand.

Rand: nach außen geneigt.

Boden: eben.

Maße (cm): Höhe 15; Durchmesser des Randes 12,6; max. Durchmesser 15,2; Durchmesser des Bodens 9,2.

7. Scheibegedrehter Becher (Gefäß 2; 742 m/2009; Abb. 7: 2)

Zustand: Rekonstruktion möglich.

Form: halbkugelig.

Oberfläche: poliert, Farbe - orange, mit Spuren von sekundärem Brand.

Rand: gerade.

Boden: abgerundet, keine Abflachung.

Ornament: kompliziert Komposition aus plastischer Wulst, eingeglätteter Linie, gestempelte und geprägte Verzierung. Maße (cm): Höhe 8,1; Durchmesser des Randes 10,2.

\section{Grab 211}

8. Bronzene zweiteilige Fibel mit umgeschlagenem Fuß (No. 2; 39 m/2015; Abb. 10: 8)

Bügel: bogenförmig, Querschnitt - Segment, 3 rechteckige Flächen am Ansatz des Fibelfußes und nahe der Spirale. Spirale: mit eiserner Achse und Ringen an beiden Enden, 12 Windungen an jeder Seite, untere doppelte Sehne. Maße (cm): gesamte Länge - 3,7; Breite des Bogens - 0,5 cм; Länge der Spirale- 4, 0.

Datierung: 2., 3. Phase der Chronologie der Gräberfelder der Cernjachov-Kultur in die ukrainische Waldsteppe nach E. L. Gorokhovski oder Ende der Stufe C2-C3 (Gorokhovski 1988, 43, 44). 
9. Bronzene zweiteilige Fibel mit umgeschlagenem Fuß (No. 2; 44 m/2015; Abb. 10: 9)

Bügel: bogenförmig, Querschnitt - Segment, 3 rechteckige Flächen am Ansatz des Fibelfußes und nahe der Spirale. Spirale: mit eiserner Achse und Ringen an beiden Enden, 12 Windungen an jeder Seite (ein Ende ist zerstört), untere doppelte Sehne.

Maße (cm): gesamte Länge 3,9; Breite des Bogen 0,5 cm; Länge der Spirale 3,6.

Datierung: 2. und 3. Phase der Chronologie der Gräberfelder der Černjachov-Kultur in die ukrainische Waldsteppe nach E. L. Gorokhovski oder Ende der Stufe C2-C3 (Gorokhovski 1988, 43, 44).

10. Einfarbige Glasperle (41 m/2015; Abb. 10: 1$)$

Form: kugelig, abgeflacht.

Durchlochung: zylindrisch.

Farbe: einfarbig, blau, durchsichtig.

Maße (cm): Länge - 1; Durchmesser - 1,8; Durchmesser der Durchlochung - 0,6.

Datierung: nicht vor dem letzten Drittel des 3. Jhs. (Gopkalo 2008, 21, 22).

11. Einfarbige Glasperle (42 m/2015; Abb. 10: 3)

Form: gerieft, 3 verbundene kugelförmige Teile.

Kanal: zylindrisch.

Farbe: dunkel-rot, undurchsichtig.

Maße (cm): Länge 1,2; Durchmesser 1; Durchmesser der Durchlochung 0,5.

12. Einfarbige Glasperle (43 m/2015; Abb. 10: 2)

Form: kugelig, abgeflacht.

Kanal: zylindrisch.

Farbe: schwarz, undurchsichtig.

Maße (cm): Länge 0,6; Durchmesser 1,1; Durchmesser der Durchlochung 0,4.

13. Einfarbige Glasperle ( 47 m/2015; Abb. 10: 4)

Form: kugelig mit Rippen.

Durchlochung: zylindsrisch

Farbe: dunkelrot, undurchsichtig.

Maße (cm): Länge 0,6; Durchmesser 1,1; Durchmesser der Durchlochung 0,4.

Datierung: nicht vor dem letzten Drittel des 3. Jhs. (Gopkalo 2008, 35).

14. Karneolperle (45 m/2015; Abb. 10: 5)

Form: mit 14 Kanten, Quader mit geschliffenen Kanten.

Durchlochung: zylindrisch.

Maße (cm): Länge 2; Querschnitt 0,7 x 1,4; Durchmesser der Durchlochung 0,3.

Datierung: letzte Drittel 3. - erste Hälfte 4. Jh. (Gopkalo 2008, 75, 76).

15. Karneolperle (46 m/2015; Abb. 10: 6).

Form: mit 14 Kanten, Quader mit geschliffenen Kanten.

Durchlochung: konisch.

Maße (cm): Länge 2, 2; Querschnitt 0,9 x 1,2; Durchmesser der Durchlochung 0,4-0,2.

Datierung: letztes Drittel 3. - erste Hälfte 4. Jh. (Gopkalo 2008, 75, 76).

16. Dreilagenkamm aus Bein (48 m/2015; Abb. 10: 10)

Form der Kopfplatte: rund.

Zähne: gerade.

Konstruktion: 7 Zahnplatten werden mit zwei Deckplatten durch 10 Bronzeniete verbunden. Auf den Griffplatten ist der Geweihkern sichtbar. Die Nieten sind aus bronzenen Platten gefertigt.

Ornament: Zirkelornament an den Deckplatten.

Maße (cm): Höhe 6,1; Höhe der Kopfplatte 3,6; Breite 11,4; Dicke 0,9.

Datierung: 1.-3. Phase der Chronologie der Gräberfelder der Černjachov-Kultur in die ukrainische Waldsteppe nach E. L. Gorokhovski oder Stufen C2-C3 (Gorokhovski 1988, 42-44).

17. Amulettanhänger (No. 3,53 m/2015; Abb. 11: 3)

Muschel der Meerschnecke Cypraea mit Loch.

Maße (cm): Länge 6,5; Breite 4; Durchmesser des Loches 0,6.

18. Amulettanhänger (No. 2, 52 m/2015; Abb. 11: 2)

Knochen (Astragal) eines Tieres der Bovidae mit dem Loch. Maße (cm): Länge 3,6; Breite 2; Durchmesser des Loches 0,6.

19. Amulettanhänger (50m/2015; Abb. 11: 4)

Hauer vom Suidae mit Loch. Maße (cm): Länge 8,8; Dicke 1,1; Durchmesser des Loches 0,4.

20. Amulettanhänger (49 m/2015; Abb. 11: 1)

Unterkiefer eines Canidae mit Loch.

Maße (cm): Länge 8,7; Breite 2,2; Durchmesser des Loches 0,4. 
21. Keramikspinnwirtel (54 m/2015; Abb. 10: 7)

Form: doppelkonisch, mit unscharfem Umbruch und konkaver Grundfläche.

Oberfläche: glatt, Farbe Grau.

Maße (cm): Höhe 1,3; Durchmesser der Grundfläche 2,0; größter Durchmesser 2,6; Durchmesser des Loches 0,7.

22. Glasschale $(40 \mathrm{~m} / 2015)$

Parameter und Beschreibung s. Anhang 1, No. 2.

23. Henkellose Kanne, scheibengedreht (Gefäß 1; 394 m/2015; Abb. 9: 1)

Zustand: vollständig.

Form: doppelkonisch, mit scharfem Umbruch und zylindrischem Hals.

Oberfläche: poliert, Farbe Grau.

Rand: abgestuft, Ende geradegebogen.

Umbruch: in der Mitte des Gefäßbauches.

Boden: abgesetzte Bodenplatte.

Ornament: plastische Wulst, Absatz zwischen Schulter und Hals.

Maße (cm): Höhe 17,8; Höhe des Halses 10,8; Durchmesser des Randes 5,5; Durchmesser des Halses 4; max. Durchmesser des Bauches 12,3; Durchmesser des Bodens 6,2.

24. Schale geschlossenen Typs (Gefäß 2; 397 m/2015; Abb. 9: 3)

Zustand: vollständig.

Form: doppelkonisch, mit scharfem Umbruch.

Oberfläche: rau, uneben, Farbe Schwarz/dunkel-grau, hell-braun/gelb. Die äußere und innere Oberfläche wird nochmals durch eine Lehmschicht bedeckt. Ein Teil dieser Schicht ist zerstört.

Rand: nach außen geneigt.

Umbruch: in der Gefäßmitte.

Boden: abgesetzte Bodenplatte.

Maße (cm): Höhe 7,6; Durchmesser des Randes 14,7; max. Durchmesser des Bauches 15,6; Durchmesser des Bodens 6,8 .

25. Schale geschlossenen Typs (Gefäß 3; 384 m/2015; Abb. 9: 2)

Zustand: Rekonstruktion möglich.

Form: doppelkonisch, mit scharfem Umbruch.

Oberfläche: poliert, Farbe - dunkelgrau/schwarz.

Rand: nach außen geneigt.

Umbruch: etwas oberhalb der Gefäßmitte.

Boden: mit Standring.

Ornament: plastischer Wulst, Absatz zwischen Schulter und Hals.

Maße (cm): Höhe 9,2; Durchmesser des Randes 18,5; max. Durchmesser des Bauches 20,1; Durchmesser des Bodens 7,0 .

26. Vase mit drei Henkeln, scheibengedreht (Gefäß 4; 938 m/2015; Abb. 9: 5)

Zustand: Rekonstruktion möglich.

Form: offener Typ, zylindrisch-konisch, mit unscharfem Umbruch.

Oberfläche: poliert, Farbe schwarz, schwach gebrannt.

Rand: eben, profiliert, D - förmig.

Umbruch: in der Mitte der Gefäßhöhe.

Henkel: profiliert, zwischen Rand und Umbruch.

Boden: mit Standring.

Ornament: komplizierte, plastischer Wulst/Absatz zwischen Schulter und Hals, auf der Schulter - „Netz“ aus eingeglätteten Linien.

Maße (cm): Höhe 18; Durchmesser des Randes 36,6; max. Durchmesser des Bauches 33,7; Durchmesser des Bodens 11,8 .

27. Scheibengedrehter Becher (Gefäß 5; 435 m/2015; Abb. 9: 4)

Zustand: in Fragmenten, Rekonstruktion unmöglich.

Oberfläche: poliert, Farbe schwarz, schwach gebrannt. 


\section{LITERATUR}

Ambroz 1966

Barkóczi 1988

Brüggler 2009

Cool 1995

Dévai 2016

Dévai 2017

Follmann-Schulz 2015

Fünfschilling 2015

Gavritukhin 1999

Gavritukhin 2017

Godłowski 1970

Gomolka-Fuchs 1999

Gopkalo 2008

Gopkalo 2011

Gorokhovski 1988

Isings 1957

Kleemann 2017

Kropotkin 1970

Kucharczyk 2007

Lazar 2003

Ljubičhev 2006

Lyubichev 2009

Lyubichev 2013

Lyubichev/Myzgin/Varacheva 2006
A. K. Ambroz: Fibuly yuha evropeiskoi chasti SSSR II v. do n. e. IV v. n. e. Moskva 1966.

L. Barkóczi: Pannonische Glasfunde in Ungarn. Budapest 1988.

M. Brüggler: Villa rustica, Glashütte und Gräberfeld. Die kaiserzeitliche und spätantike Siedlungsstelle HA 132 im Hambacher Forst. In: Rheinische Ausgrabungen 63. Mainz 2009, 83-88.

H. E. M. Cool: Glass vessels of the fourth and early fifth century in Roman Britain. In: D. Foy (ed.): Le verre de l'antiquite Tardive et haut moyen age. Val d'Oise 1995, 11-26.

K. Dévai: Glass vessels from Late Roman times found in Pannonia. Acta Archaeologica Academiae Scientiarum Hungaricae 67, 2016, 255-286.

K. Dévai: Glass vessels from Late Roman graves in the Hungarian part of the Roman province Pannonia. In: S. Wolf/A. Pury-Gysel (eds.): Association Internationale pour l'Histoire du Verre. Annales du 20e Congrés 2015. Romont 2017, 222-229.

A. -B. Follmann-Schulz: The Hambach glass production in the Late Roman period In: J. Bayley/I. Freestone/C. Jackson (eds.): Glass of the Roman world. Oxford 2015, 24-32.

S. Fünfschilling: Die römischen Gläser aus Augst und Kaiseraugst. Augst 2015.

I. O. Gavritukhin: Khronologicheskie indikatory finala chernyakhovskoy kultury. In: M. Levada (ed.): Sto let chernyakhovskoy kulture. Kiev 1999, 48-86.

I. Gavritukhin: Glass vessels of the final of the Chernyakhov culture. In: $\mathrm{Na}$ hranicích impéria. Extra fines imperii. Jaroslavu Tejralovi $k$ 80. narozeninám. Brno 2017, 83-109.

K. Godłowski: The chronology of the Late Roman and Early Migration periods in Central Europe. Kraków 1970.

G. Gomolka-Fuchs: Gläser der Sîntana de Mureş-Černjachov-Kultur aus Rumänien und der Republik Moldavien. In: G. Gomolka-Fuchs (ed.): Die Sîntana de Mureş-Černjachov-Kultur. Akten des Internationalen Kolloquiums in Caputh vom 20. bis. 24. October 1995. Bonn 1999, 129-142.

O. V. Gopkalo: Busy i podveski chernyakhovskoi kultury. Kiev 2008.

O. V. Gopkalo: Muzhskoy i zhenskiy chernyakhovskiy kostyum (po dannym pogrebeniy s antropologicheskimi opredeleniyami). Stratum plus 4, 2011, $1-29$.

E. L. Gorokhovski: Hronologija chernjahovskih mogilnikov lesostepnoj Ukrainy. In: Trudy V Mezhdunarodnogo kongressa arheologov-slavistov 4. Kiev 1988, 34-46.

C. Isings: Roman glass from dated finds. Archaeologica Traiectina II. Groningen Djakarta 1957.

J. Kleemann: Die Ausgrabungen des Städtischen Museums Marienburg im Gräberfeld Malbork-Wielbark Fundstelle 1 in den Jahren 1927-1932, 1934 und 1936. Lublin 2017.

V. V. Kropotkin: Rimskie importnye izdeliya v Vostochnoy Evrope. Svod arkheologicheskikh istochnikov D1-27. Moskva 1970.

R. Kucharczyk: Late Roman/Early Byzantine glass from the basilica in Marea. Polish Archaeology in the Mediterranean 17, 2007, 70-74.

I. Lazar: Rimsko steklo Slovenije/Roman glass of Slovenije. Ljubljana 2003.

M. V. Ljubičhev: Vojtenki 1. Eine Siedlung der Cernjachov-Kultur in der Ostukraine. Eurasia Antiqua 12, 2006, 269-288.

M. V. Lyubichev: Pogrebenie s rimskim importom na mogilnike chernyakhovskoy kultury Voytenki (Vostochnaya Ukraina). In: K. V. Myzgin (ed.): Ostrogothica. Arkheologiya Tsentralnoy $i$ Vostochnoy Evropy pozdnerimskogo vremeni i Epokhi Velikogo pereseleniya narodov. Kharkov 2009, 72-90.

M. Lyubichev: The Secondary Used Artifact in Grave 41 of the Chernyakhov Cemetery of Voytenki. In: I. Khrapunov/F. A. Stylegar (eds.): Inter ambo maria. Northeren Barbarians from Scandinavia towards the Black sea. Kristiansand-Simferopol 2013, 236-248.

M. V. Lyubichev/K. V. Myzgin/K. G. Varacheva: Otchet o rabote Germano-Slavyanskoy ekspeditsii Kharkovskogo natsionalnogo universiteta imeni V. N. Karazina v 2005 godu. Kharkov 2006. 
Lyubichev/Myzgin/Varacheva 2007

Lyubichev/Myzgin/Varacheva 2008

Lyubichev/Myzgin/Varacheva 2009

Lyubichev/Myzgin/Varacheva 2010

Lyubichev/Myzgin/Varacheva 2011

Lyubichev/Myzgin/Varacheva 2012

Lyubichev/Myzgin/Varacheva 2013

Lyubichev u. a. 2014

Lyubichev u. a. 2015

Lyubichev u. a. 2016

Lyubichev u. a. 2017

Lyubichev u. a. 2018

Lyubichev/Schultze 2018

Lyubichev/Schultze/Gella 2019

Magomedov 2001

Magomedov 2015

Natuniewicz-Sekuła/Kontny 2010

Natuniewicz-Sekuła/Okulicz-Kozaryn 2011

Nekrasova 1985

Nekrasova 2006
M. V. Lyubichev/K. V. Myzgin/K. G. Varacheva: Otchet o rabote Germano-Slavyanskoy arkheologicheskoy ekspeditsii Kharkovskogo natsionalnogo universiteta imeni V. N. Karazina v 2006 godu. Kharkov 2007.

M. V. Lyubichev/K. V. Myzgin/K. G. Varacheva: Otchet o rabote Germano-Slavyanskoy arkheologicheskoy ekspeditsii Kharkovskogo natsionalnogo universiteta imeni V. N. Karazina v 2007 godu. Kharkov 2008.

M. V. Lyubichev/K. V. Myzgin/K. G. Varacheva: Otchet o rabote Germano-Slavyanskoy arkheologicheskoy ekspeditsii Kharkovskogo natsionalnogo universiteta imeni V. N. Karazina v 2008. Kharkov 2009.

M. V. Lyubichev/K. V. Myzgin/K. G. Varacheva: Otchet o rabote Germano-Slavyanskoy arkheologicheskoy ekspeditsii Kharkovskogo natsionalnogo universiteta imeni V. N. Karazina v 2009 godu. Kharkov 2010.

M. V. Lyubichev/K. V. Myzgin/K. G. Varacheva: Otchet o rabote Germano-Slavyanskoy arkheologicheskoy ekspeditsii Kharkovskogo natsionalnogo universiteta imeni V. N. Karazina v 2010. Kharkov 2011.

M. V. Lyubichev/K. V. Myzgin/K. G. Varacheva: Otchet o rabote Germano-Slavyanskoy arkheologicheskoy ekspeditsii Kharkovskogo natsionalnogo universiteta imeni V. N. Karazina v 2011(nomer ne prisvoen). Kharkov 2012.

M. V. Lyubichev/K. V. Myzgin/K. G. Varacheva: Otchet o rabote Germano-Slavyanskoy arkheologicheskoy ekspeditsii Kharkovskogo natsionalnogo universiteta imeni V. N. Karazina v 2012. Kharkov 2013.

M. V. Lyubichev/K. V. Myzgin/K. G. Varacheva/E. Schultze: Otchet o rabote Germano-Slavyanskoy arkheologicheskoy ekspeditsii Kharkovskogo natsionalnogo universiteta imeni V. N. Karazina v 2013. Kharkov 2014.

M. V. Lyubichev/K. V. Myzgin/K. G. Varacheva/E. Schultze: Otchet o rabote Germano-Slavyanskoy arkheologicheskoy ekspeditsii Kharkovskogo natsionalnogo universiteta imeni V. N. Karazina v 2014 (nomer ne prisvoen). Kharkov 2015.

M. V. Lyubichev/K. V. Myzgin/K. G. Varacheva/E. Schultze: Otchet ob issledovanii arkheologicheskogo kompleksa pozdnerimskogo vremeni Voytenki v 2015 (nomer ne prisvoen). Kharkov 2016.

M. V. Lyubichev/K. V. Myzgin/K. G. Varacheva/E. Schultze/D. A. Filatov: Otchet o rabote Germano-Slavyanskoy arkheologicheskoy ekspeditsii Kharkovskogo natsionalnogo universiteta imeni V. N. Karazina v 2016 (nomer ne prisvoen). Kharkov 2017.

M. V. Lyubichev/D. A. Filatov/K. V. Myzgin/E. Schultze/K. G. Varacheva/ V. V. Rusnak: Otchet o rabote Germano-Slavyanskoy arkheologicheskoy ekspeditsii Kharkovskogo natsionalnogo universiteta imeni V. N. Karazina v 2017 (nomer ne prisvoen). Kharkov 2018.

M. V. Lyubichev, E. Schultze: Zu einer Gruppe von Körpergräbern im nordwestlichen Bereich des Gräberfeldes der Černjachov-Kultur, Vojtenki: Fragen zur Chronologie. In: M. Lyubichev/K. Myzgin (eds.): Khronologiya i monetnye nakhodki pozdnerimskogo vremeni i epokhi Velikogo pereseleniya narodov. Aktualnye arkheologicheskie issledovaniya $v$ Tsentralnoy $i$ Vostochnoy Evrope. OstrogothicaSerie (Hefte) Heft 3. Kharkov 2018, 121-145.

M. V. Lyubichev/E. Schultze/E. I, Gella: Pozdnie pogrebeniya mogilnika Voytenki i poslednyaya faza chernyakhovskoy kultury v lesostepi mezhdu Dneprom i Severskim Dontsom. In: O. V. Petrauskas/O. V. Hopkalo/S. A. Horbanenko (eds.): Starozhytnosti varvarskykh plemen u pershii polovyni I tys. n. e.: do 90-richchia V. D. Barana Oium 6. Kiev 2019, 13-49.

B. V. Magomedov: Chernyakhovskaya kultura. Problema etnosa. Lublin 2001.

B. V. Magomedov: Keramicheskie pryaslitsa chernyakhovskoy kultury. Kiev 2015.

M. Natuniewicz-Sekuła/B. Kontny: "Jeździec bez głowy” et alii, czyli znaleziska ostróg z cmentarzyska kultury wielbarskiej w Weklicach. In: A. Urbaniak/R. Prochowicz/I. Jakubczyk/M. Levada/J. Schuster (eds.): Terra Barbarica. Monumenta Archaeologica Barbarica. Series Gemina Tomus II. Łodź-Warszawa 2010, 333-345.

M. Natuniewicz-Sekuła/J. Okulicz-Kozaryn: Weklice. A cemetery of Wielbark Culture on the Eastern Margin of Vistula Delta (Excavations 1984-2004). Warszawa 2011.

H. M. Nekrasova: Okhoronni rozkopky cherniakhivskoho mohylnyka poblyzu m. Sumy. Arkheolohiia 50, 1985, 75-80.

A. N. Nekrasova: Pamyatniki chernyakhovskoy kultury Dneprovskogo Levoberezhya. In: Goty i Rim. Sbornik nauchnykh statey. Kiev 2006, 87-200. 
Nikitina 1969

Petrauskas 2002

Petrauskas 2003

Petrauskas 2009

Petrauskas 2014

Petrauskas 2016

Petrauskas 2017

Petrauskas/Pasternak 2003

Rafalovich 1986

Rau 1972

Reida/Heiko/Sapiehin 2015

Reida/Heiko/Sapiehin 2018

Rumyantseva 2016

Rumyantseva $2017 a$

Rumyantseva 2017 b

Rumyantseva/Belikov 2017

Rumyantseva/Lyubichev/Trifonov 2018

Rumyantseva/Shcherbakov 2016
G. F. Nikitina: Grebni chernyakhovskoy kultury. Sovetskaya Arkheologiya 1, 1969, 147-160.

O. V. Petrauskas: Typy arkheolohichnykh kompleksiv trupospalen cherniakhivskoi kultury. Arkheolohiia 3, 2002, 40-65.

O. V. Petrauskas: Die Gräberfelder der Černjachov-Kultur von Kosanovo und Gavrilovka - eine vergleichende Studie zu Chronologie, Bestattungssitten und ethnokulturellen Besonderheiten. Bericht der Römisch-Germanischen Kommission 84, 2003, 224-362.

O. V. Petrauskas: Chas poiavy ta deiaki osoblyvosti rozvytku trupopokladen iz zakhidnoiu oriientatsiieiu $\mathrm{v}$ cherniakhivskii kulturi (za danymy mohylnykiv Ukrainy). In: Ostrogothica. Arkheolohyia Tsentralnoi y Vostochnoi Evropy pozdnerimskoho vremens i Epokhs Velykoho pereselenyia narodov. Kharkov 2009, 186-215.

O. V. Petrauskas: Razrushennye pogrebeniya na mogilnikakh chernyakhovskoy kultury Podneprovya: analiz arkheologicheskoy struktury. In: H. Yu. Ivakin (ed.): Vid venediv do Rusi. Kiev 2014, 125-152.

O. V. Petrauskas: Skliani posudyny typu Eggers/230 v cherniakhivskii kulturi: khronolohichnyi aspekt. In: O. V. Petrauskas/O. V. Hopkalo/S. A. Horbanenko (eds.): Cherniakhivska kultura. Oium 5. Kiev 2016, 89-106.

O. V. Petrauskas: Übergang von der Stufe C2 zu C3 in der Černjachov-Kultur (auf dem Beispiel der Glasschalen mit Ringboden). Ephemeris Napocensis 27, 2017, 123-154.

O. V. Petrauskas/V. V. Pasternak: Skliani posudyny mohylnyka cherniakhivskoi kultury Velyka Buhaivka v Serednomu Podniprovi. Arkheolohiia 4, 2003, 65-74.

I. A. Rafalovich: Dancheny. Mogilnik chernyakhovskoy kultury III-IV vv. n. e. Kishinev 1986

G. Rau: Körpergräber mit Glasbeigaben des 4. nachchristlichen Jahrhunderts im Oder-Weichsel-Raum. Acta Praehistorica et Archaeologica 3, 1972, 109-215.

R. M. Reida/A. V. Heiko/S. V. Sapiehin: «Potoibichnyi banket»: nabory hlekiv u pokhovanniakh cherniakhivskoi kultury. Arkheolohiia 1, 2015, 30-39.

R. M. Reida/A. V. Heiko/S. V. Sapiehin: Deiaki khronoindykatory druhoi polovyny IV-V st. z Shyshatskoho mohylnyka. In: M. Lyubichev/K. Myzgin (eds.): Khronologiya i monetnye nakhodki pozdnerimskogo vremeni i epokhi Velikogo pereseleniya narodov. Aktualnye arkheologicheskie issledovaniya v Tsentralnoy $i$ Vostochnoy Evrope. Ostrogothica-Serie (Hefte) Heft 3. Kharkov 2018, $111-120$

O. S. Rumyantseva: Litoe steklo s poseleniya chernyakhovskoy kultury Komarov: prestizhnaya posuda ili syre steklodelatelnoy masterskoy? Kratkie soobshcheniya Instituta Arkheologii 245, 2016, 299-315.

O. S. Rumyantseva: Steklodelie za limesom: novye dannye ob organizatsii proizvodstva i khronologii masterskoy v Komarove. Stratum plus 4, 2017, $141-164$

O. S. Rumyantseva: Riflenye formy i nakhodki riflenogo stekla s poseleniya chernyakhovskoy kultury Komarov: k voprosu o khronologii i vidakh produktsii stekolnoy masterskoy. In: I. O. Gavritukhin/N. V. Lopatin/A. M. Oblomskiy (eds.): Ranneslavyanskiy mir. Arkheologiya slavyan i ikh sosedey 19. Moskva 2017, 193-199.

O. Rumyantseva/S. A. Belikov: Late Roman workshop at Komarov (Middle Dniester) and the problem of the origin of 'barbarian' facet cut beakers. In: S. Wolf/A. Pury-Gysel (eds.): Association Internationale pour l'Histoire du Verre Annales du 20e Congrés 2015. Romont 2017, 257-264.

O. S. Rumyantseva/M. V. Lyubichev/A. A. Trifonov: Khimicheskiy sostav stekla arkheologicheskogo kompleksa Voytenki 1 i proiskhozhdenie sosudov chernyakhovskoy kultury (predvaritelnye itogi issledovaniy) In: M. Lyubichev/K. Myzgin (eds.): Khronologiya i monetnye nakhodki pozdnerimskogo vremeni i epokhi Velikogo pereseleniya narodov. Aktualnye arkheologicheskie issledovaniya $v$ Tsentralnoy $i$ Vostochnoy Evrope. Ostrogothica-Serie (Hefte) Heft 3. Kharkov 2018, 182-208.

O. S. Rumyantseva/I. B. Shcherbakov: Steklo-syrets s poseleniya Komarov na Srednem Dnestre: khimicheskiy sostav i dannye o kharaktere i khronologii steklodelatelnogo kompleksa pozdnerimskogo vremeni. Stratum plus 4, 2016, 203-217. 
Rutkovskaya 1979

Rütti 1991

Schultze/Lyubichev 2012

Schultze/Lyubichev 2015

Schultze/Lyubichev 2017

Shchukin/Shcherbakova 1986

Shishkin 1999

Stawiarska 1999

Stawiarska 2014

Tejral 1986
L. M. Rutkovskaya: Arkheologicheskie pamyatniki IV-VI vv. v rayone Kremenchugskogo morya (Ukraina). Slovenská archeológia 27, 1979, 317-362.

B. Rütti: Die römischen Gläser aus Augst und Kaiseraugst. Augst 1991.

E. Schultze/M. Lyubichev: Khronologiya mogilnika chernyakhovskoy kultury Voytenki 1 (Vostochnaya Ukraina po materialam raskopok 2005-2009). In: A. M. Vorontsov/I. O. Gavritukhin (eds.): Lesnaya i lesostepnaya zony Vostochnoy Evropy $v$ epokhi rimskikh vliyaniy $i$ Velikogo pereseleniya narodov. Tula 2012, 409-463.

E. Schultze/M. V. Lyubichev: Kultovyy sloy mogilnika chernyakhovskoy kultury Voytenki (Vostochnaya Ukraina). Spektr nakhodok i vozmozhnosti interpretatsii. In: A. M. Vorontsov/I. O. Gavritukhin (eds.): Lesnaya i lesostepnaya zony Vostochnoy Evropy v epokhi rimskikh vliyaniy $i$ Velikogo pereseleniya narodov. Konferentsiya 4. Chast 1. Tula 2015, 231-255.

E. Schultze/M. V. Lyubichev: A Cremation Grave of the Chernyakhov Culture with Spurs and Knop Bow Fibula (Bügelknopffibel): Regional Context and Interregional Comparison. In: M. Matera/R. Karasiewicz-Szczypiorski (eds.): The Crimea and the Nothern Black Sea Coast in Archaeological Research 1956-2013. Swiatowit Supplement. Series C. Pontica et Caucasica 1. Warsaw 2017, 277-294. n

M. B. Shchukin/T. A. Shcherbakova 1986: K khronologii mogilnika Dancheny. In: I. A. Rafalovich Dancheny (ed.): Mogilnik chernyakhovskoy kultury III-IV vv. n. e. Kishinev 1986, 177-208.

R. G. Shishkin: Klassifikatsiya i tipologiya trekhsloynykh grebney chernyakhovskoy kultury. In: M. Levada (ed.): Sto rokiv vyvchennia kultury poliv pokhovan na Ukraini. Kiev 1999, 43-48.

T. Stawiarska: Naczynia szklane okresu rzymskiego z terenu Polski. Warszawa 1999.

T. Stawiarska: Roman and Early Byzantine glass from Romania and Northern Bulgaria. Bibliotheca Antiqua 24. Warsaw 2014.

J. Tejral: Fremde Einflüsse und kulturelle Veränderungen nördlich der mittleren Donau zu Beginn der Völkerwanderungszeit. Archaeologia Baltica VII, 1986, 175-238.

\title{
Glass bowls of Weklice type from graves 110 and 211 of Vojtenki 1 Late Roman - Early Great migration period burial ground
}

\author{
Vladyslav Shchepachenko
}

Summary

Current publication presents an attempt to find the closest analogies to the glass bowls from graves 110 and 211 on Vojtenki 1 burial ground (Eastern Ukraine). As result, it had managed to find out, that the similar vessels came from Northern Poland and Lower Danubian region. These analogies are vessels from Weklice, Poland (burial 82) and Danceni, Republic Moldova (burial 169). T. Stawiarska was identified them as "Weklice" type. Distinctive and peculiar morphological features of each of the bowls indicate that the Weklice type vessels are unlikely to have a common origin. However, despite these differences, similar shape, the same techniques for processing a rim and a similar ornament composition allow us to rank these bowls to type of product. Some researchers have associated "hot decoration" way of glassware production with the provincial-Roman glassworking technology. That is why, we could assumed, that Weklice type glass bowls have a provincial-Roman origin. The evolution of the glassware forms in Roman world allows us to establish the time of their production. It is limited to the period between the second third of the $3^{\text {rd }}$ century - the end of $3 \mathrm{rd} /$ beginning of $4^{\text {th }}$ centuries. Lower chronological frame corresponds to the time of appearance of such products in Barbaricum, and the upper one, to the time of disappearance of glassware with fire-rounded rim in a number of border provinces of the Empire. Nevertheless, the dating of burials 110 and 211 from Vojtenki 1, shows that such products continue to exist in Barbaricum for a rather long time. It also should be noted, that at the Vojtenki 1 burial ground, such bowls are found only in high or special social status female graves. Moreover, the same type of vessel appears in burials with different burial rite as a grave good. 
Fig. 1. A - glass bowls type Weklice in Barbaricum; B - plan of the archaeological complex Vojtenki 1. Legend: gray area of the Sântana de Mureş-Chernyahov culture; 1 - Vojtenki 1; 2 - Danceni; 3 - Weklice.

Fig. 2. Glass bowls type Weklice. 1 - Vojtenki 1, grave 110 (Supplement 1, No. 1); 2 - Vojtenki 1, grave 211 (Supplement 1 , No. 2); 3 - Weklice, grave 82 (Supplement 1, No. 3; according to Natuniewicz-Sekuła/Okulicz-Kozaryn 2011); 4 -Danceni, grave 169 (Supplement 1, No. 4; according to Rafalovich 1986). Photo by V. Vornic. Without scale: 4.

Fig. 3. Chronology of hemispherical glass bowls with fire-rounded and unprocessed/polished rim in Barbaricum and some provinces of the Roman Empire. Legend: black - exactly determined period; gray - probable period.

Fig. 4. Glass bowls type Weklice and their analogies. 1 - Vojtenki 1, grave 110; 2 - Vojtenki 1, grave 211; 3 - Marea; 4 - Weklice, grave 82; 5 - Dănceni, grave 169; 6 - Nizhnij Strutin; 7 - Rudka; 8 - Chernyahov, grave 88; 9 - MalborkWielbark, grave 1180; 10 - glass bowl type Eggers 200 and beakers type Eggers 188-190. 3 - according to Kucharczyk 2007; 4 - according to Natuniewicz-Sekuła/Okulicz-Kozaryn 2011; 5 - according to Rafalovich 1986; 6 - according to Kropotkin 1970; 7, 8 - according to Rau 1972; 9 - according to Kleemann 2017. 5-8, 9 - analogies according to T. Stawiarska; 5, 6, 10 - analogies according to M. B. Shchukin and T. A. Shcherbakova. Scale: a - 1, 2, 4; b-6-8; without scale - 3, $5,9,10$.

Fig. 5. The plan of Vojtenki 1 burial ground. Part which was investigated in 2005-2017.

Fig. 6.1 - the plan; 2 - the profile of grave 110 of Vojtenki 1 burial ground.

Fig. 7. Grave goods from the grave 110 of Vojtenki 1 burial ground. 1 - wheel-made pot of baked clay (Supplement 2, No. 6); 2 - wheel-made beaker of baked clay (Supplement 2, No. 7); 3 - carnelian bead (Supplement 2, No. 4); 4 - bronze rivet of multi-part antler comb (Supplement 2, No. 5); 5 - spindle whorl of baked clay (Supplement 2, No. 2); 6 - spindle whorl of baked clay (Supplement 2, No. 1).

Fig. 8. The plan of grave 211 of Vojtenki 1 burial ground.

Fig. 9. Grave goods from the grave 211 of Vojtenki 1 burial ground. 1 - wheel-made jug of baked clay without handles (Supplement 2, No. 23); 2 - wheel-made closed bowl of baked clay (Supplement 2, No. 24); 3 - wheel-made closed bowl of baked clay (Supplement 2, No. 25); 4 - wheel-made beaker of baked clay (Supplement 2, No. 27); 5 - wheel-made vase of baked clay with three handles (Supplement 2, No. 26).

Fig. 10. Grave goods from the grave 211 of Vojtenki 1 burial ground. 1 - monochrome glass bead (Supplement 2, No. 10); 2 - monochrome glass bead (Supplement 2, No. 12); 3 - monochrome glass bead (Supplement 2, No. 11); 4 - monochrome glass bead (Supplement 2, No. 13); 5 - carnelian bead (Supplement 2, No. 14); 6 - carnelian bead (Supplement 2, No. 15); 7 - spindle whorl of baked clay (Supplement 2, No. 21); 8 - bronze two-piece fibula with returned foot (Supplement 2, No. 8); 9 - bronze two-piece fibula with returned foot (Supplement 2, 9); 10 - multi-part antler comb (Supplement 2, No. 16).

Fig. 11. The set of the amulet-pendants from the graves 211 (1-4) and 68 (5) of Vojtenki 1 burial ground. 1 - amulet-pendant (Supplement 2, No. 20); 2 - amulet-pendant (Supplement 2, No. 18); 3 - amulet-pendant (Supplement 2, No. 17); 4 - amulet-pendant (Supplement 2, No. 19). Without scale: 5.

Tab. 1. The set of the amulet-pendants from the graves of Vojtenki 1 burial ground.

Translated by E. Schultze, M. V. Lyubichev

Shchepachenko Vladyslav I. - bachelor

Historical Faculty of Vasil Karazin National University

Laboratory assistant

Educational-research laboratory of the German-Slavonic

Archaeological expedition of Vasil Karazin National University

Maidan Svobody 4

UA - 61022 Kharkiv

schepachenko@karazin.ua 